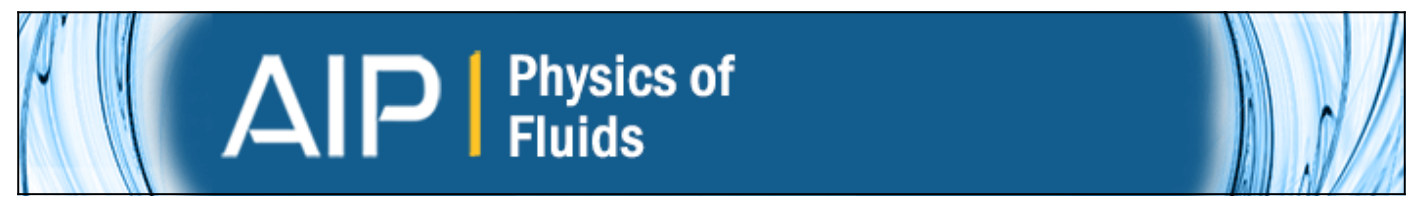

\title{
Inertial convection in a rotating narrow annulus: Asymptotic theory and numerical simulation
}

Keke Zhang, Xinhao Liao, and Dali Kong

Citation: Physics of Fluids 27, 106604 (2015); doi: 10.1063/1.4934527

View online: http://dx.doi.org/10.1063/1.4934527

View Table of Contents: http://scitation.aip.org/content/aip/journal/pof2/27/10?ver=pdfcov

Published by the AIP Publishing

\section{Articles you may be interested in}

Generation and maintenance of bulk turbulence by libration-driven elliptical instability

Phys. Fluids 27, 066601 (2015); 10.1063/1.4922085

Experimental and numerical study of buoyancy-driven single bubble dynamics in a vertical Hele-

Shaw cell

Phys. Fluids 26, 123303 (2014); 10.1063/1.4903488

Centrifugally driven thermal convection in a rotating porous cylindrical annulus

Phys. Fluids 25, 044104 (2013); 10.1063/1.4802050

Direct numerical simulation of transitions towards structural vacillation in an air-filled, rotating, baroclinic annulus

Phys. Fluids 20, 044107 (2008); 10.1063/1.2911045

Transition to turbulence in a tall annulus submitted to a radial temperature gradient

Phys. Fluids 19, 054101 (2007); 10.1063/1.2721756

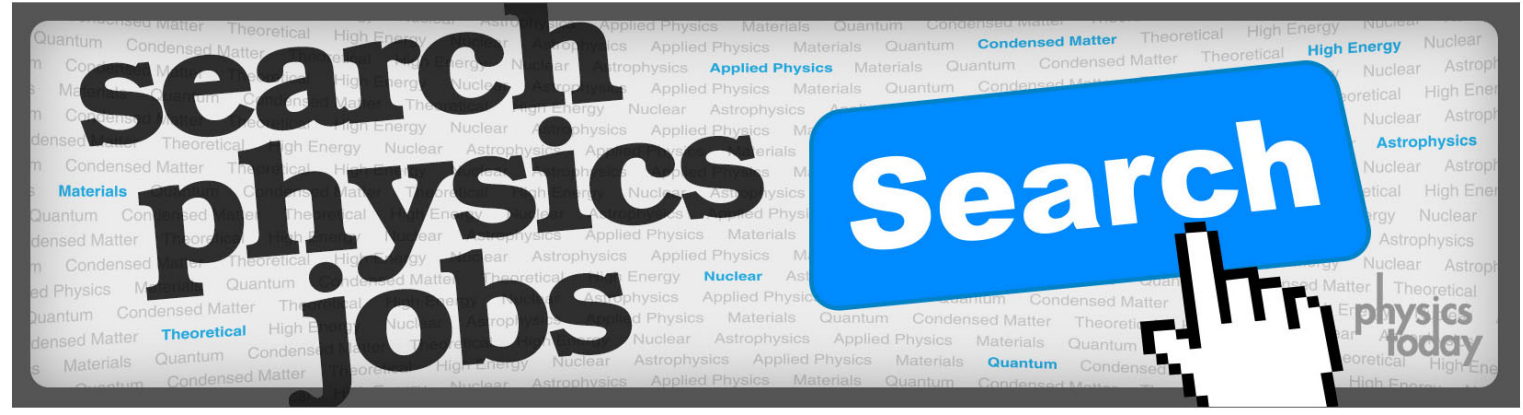




\title{
Inertial convection in a rotating narrow annulus: Asymptotic theory and numerical simulation
}

\author{
Keke Zhang, ${ }^{1,2}$ Xinhao Liao, ${ }^{3}$ and Dali Kong ${ }^{1}$ \\ ${ }^{1}$ Center for Geophysical and Astrophysical Fluid Dynamics, University of Exeter, \\ Exeter, United Kingdom \\ ${ }^{2}$ Lunar and Planetary Science Laboratory, Macau University of Science and Technology, \\ Macau, China \\ ${ }^{3}$ Key Laboratory of Planetary Sciences, Shanghai Astronomical Observatory, \\ Chinese Academy of Sciences, Shanghai 200030, China
}

(Received 24 April 2015; accepted 11 September 2015; published online 28 October 2015)

\begin{abstract}
An important way of breaking the rotational constraint in rotating convection is to invoke fast oscillation through strong inertial effects which, referring to as inertial convection, is physically realizable when the Prandtl number $\operatorname{Pr}$ of rotating fluids is sufficiently small. We investigate, via both analytical and numerical methods, inertial convection in a Boussinesq fluid contained in a narrow annulus rotating rapidly about a vertical symmetry axis and uniformly heated from below, which can be approximately realizable in laboratory experiments [R. P. Davies-Jones and P. A. Gilman, "Convection in a rotating annulus uniformly heated from below," J. Fluid Mech. 46, 65-81 (1971)]. On the basis of an assumption that inertial convection at leading order is represented by a thermal inertial wave propagating in either prograde or retrograde direction and that buoyancy forces appear at the next order to maintain the wave against the effect of viscous damping, we derive an analytical solution that describes the onset of inertial convection with the non-slip velocity boundary condition. It is found that there always exist two oppositely traveling thermal inertial waves, sustained by convection, that have the same azimuthal wavenumber, the same size of the frequency, and the same critical Rayleigh number but different spatial structure. Linear numerical analysis using a Galerkin spectral method is also carried out, showing a quantitative agreement between the analytical and numerical solutions when the Ekman number is sufficiently small. Nonlinear properties of inertial convection are investigated through direct three-dimensional numerical simulation using a finite-difference method with the Chorin-type projection scheme, concentrating on the liquid metal gallium with the Prandtl number $\operatorname{Pr}=0.023$. It is found that the interaction of the two counter-traveling thermal inertial waves leads to a timedependent, spatially complicated, oscillatory convection even in the vicinity of the onset of inertial convection. The nonlinear properties are analyzed via making use of the mathematical completeness of inertial wave modes in a rotating narrow annulus, suggesting that the laminar to weakly turbulent transition is mainly caused by the nonlinear interaction of several inertial wave modes that are excited and maintained by thermal convection at moderately supercritical Rayleigh numbers. (ㅇ 2015 AIP Publishing LLC. [http://dx.doi.org/10.1063/1.4934527]
\end{abstract}

\section{INTRODUCTION}

Oscillatory fluid motion restored by the Coriolis force, which is usually referred to as inertial waves, is ubiquitous in rotating fluid systems. Inertial waves can be excited and sustained, for example, by precession, ${ }^{1-3}$ by thermal convection, ${ }^{4-6}$ by planetary libration, ${ }^{7}$ and by differential rotation. ${ }^{8}$ Understanding how inertial waves in rotating systems are excited and maintained by thermal convection helps elucidate the nature of highly complicated convective flows in astrophysical fluid systems that are marked by very small size of the Prandtl number. ${ }^{9}$ 
Rotating convection, such as convection in a rotating Rayleigh-Bénard layer heated from below, is primarily characterized by the three dimensionless parameters: the Prandtl number $\mathrm{Pr}$ measuring the relative importance between the momentum and heat diffusions, the Ekman number $E$ relating to the rotation rate of the system, and the Rayleigh number $R a$ proportional to buoyancy forces driving convection. A fundamentally important feature of rotating convection is that the rotational effect is strongly stabilizing and, thus, the associated rotational constraint must be broken in order that convection can take place. ${ }^{10}$ There exist two profoundly different ways of breaking the rotational constraint in the weakly nonlinear state of rotating convection. The first way is to invoke large viscous forces in connection with the following asymptotic scaling for steady or slowly oscillatory convection: ${ }^{10}$

$$
\frac{L}{d} \sim(E)^{1 / 3} \text { for } 0<E \ll 1,
$$

where $L$ is the typical horizontal scale of convective flow in the bulk of the fluid and $d$ denotes the scale of the fluid container. This type of convection will be referred to as viscous convection which is physically realizable (i.e., representing the most unstable mode of convection) when the Prandtl number $\operatorname{Pr}$ of a rotating fluid, such as water, is sufficiently large. Viscous convection is energetically expensive and requires a large Rayleigh number,

$$
(R a)_{c} \sim\left(\frac{1}{E}\right)^{4 / 3} \text { for } 0<E \ll 1,
$$

where $(R a)_{c}$ denotes the critical Rayleigh number, to maintain. The corresponding convective motion would be steady or slowly oscillatory such that its inertial effect does not enter into the leading-order problem. ${ }^{10,11}$ The role of viscosity in viscous convection is inverted: instead of being purely dissipative, it provides sufficiently large frictional forces to offset the part of the Coriolis force to initiate convection. The properties of viscous convection have been extensively studied in various geometries with a huge existing literature. ${ }^{10-15}$

The second way of breaking the rotational constraint is to invoke fast oscillation through the inertial effect, which is energetically less expensive and will be referred to as inertial convection. ${ }^{4}$ It is physically realizable (i.e., representing the most unstable mode of convection) when the Prandtl number $\operatorname{Pr}$ of a rotating fluid, such as liquid gallium, is sufficiently small such that the inertial effects are dominant in the dynamics of rotating convection. The onset of inertial convection is chiefly marked by the following three characters: (i) inertial wave carries the temperature and associated density differences passively and the buoyancy force maintains convection against the viscous dissipation that primarily takes place in the thin viscous boundary layer in the case of the no-slip velocity condition; (ii) the role of viscosity, in contrast to that in viscous convection, is purely dissipative and, hence, (iii) the typical scale $L$ of inertial convection in the bulk of the fluid is comparable to that of the fluid container. It follows that the fundamental asymptotic scaling for inertial convection is

$$
\frac{L}{d} \sim 1 \text { for } 0<E \ll 1,
$$

with the critical Rayleigh number $(R a)_{c}$ required for initiating convection given by

$$
(R a)_{c} \sim\left(\frac{1}{E}\right)^{1 / 2} \text { for } 0<E \ll 1,
$$

if the no-slip condition is adopted. ${ }^{5}$ In contrast to viscous convection, the problem of inertial convection has received relatively less attention with a relatively small existing literature: Zhang ${ }^{4,5}$ first revealed the physical preference of inertial convection in the fluids of small Prandtl number and derived an asymptotic analytical solution for the onset of inertial convection in spherical geometry; Zhang and Roberts ${ }^{16}$ extended the spherical analysis to that for a rotating Rayleigh-Bénard layer; Busse and Simitev ${ }^{6}$ carried out a numerical study of spherical inertial convection; Aurnou and Olson ${ }^{17}$ performed an experimental study of inertial convection in a rotating Rayleigh-Bénard layer using the liquid gallium; and the recent laboratory experiment ${ }^{18,19}$ concentrates on the scaling law of strongly turbulent states with $(R a) /(R a)_{c} \gg \mathrm{O}(10)$ for the liquid gallium. 
Motivated by its geophysical and astrophysical application, Davies-Jones and Gilman ${ }^{20}$ studied the problem of rotating convection in a narrow annulus uniformly heated from below. By assuming that the gap-width of an annulus is small in comparison with its radius, they neglected the curvature effect of a narrow annulus via making the small-gap approximation, which will be referred to as an annular channel. There are several advantages in adopting this rotating convection model. First, the configuration of an annular channel can be approximately realizable in laboratory experiments. ${ }^{12,20}$ Second, the mathematical degeneracy characterizing an unbounded Rayleigh-Bénard layer is removed by the presence of two lateral sidewalls. Third, the local Cartesian coordinates, in contrast to spherical or cylindrical polar coordinates, provide the clarity and simplicity for mathematical analysis. Busse ${ }^{21}$ studied the problem of weakly nonlinear viscous convection in a rotating annular channel by assuming that the temperature perturbation is independent of the coordinate across the two sidewalls of the channel, which always leads to the preference of stationary convection. The numerical study ${ }^{22,23}$ focused on the wall-localized, slowly oscillatory viscous convection in a rotating annular channel. By solving the problem of rotating convection in a narrow annulus without making the small-gap approximation, Li et al. ${ }^{24}$ demonstrated the key properties of viscous convection in a rotating narrow annulus with curvature effects largely resemble those in an annular channel without having curvature effects.

The primary objective of the present study is to understand, through both asymptotic and numerical analysis, the properties of linear and moderately nonlinear inertial convection with small Prandtl numbers, such as liquid gallium, in a rotating annular channel uniformly heated from below with the non-slip boundary conditions. In what follows we shall begin by presenting the governing equations of the problem and the relevant boundary conditions in Sec. II. An asymptotic analysis deriving the analytical solution for the onset of inertial convection in a rapidly rotating annular channel is presented in Sec. III. It is fortunate that, upon recognizing and utilizing the physical nature of inertial convection, we are able to derive an explicitly analytical formula in closed form that correctly and accurately describes the key features of inertial convection in rapidly rotating annular channels. The numerical simulations for both linear and nonlinear solutions of inertial convection are discussed in Sec. IV. In the linear numerical analysis, we demonstrate that a satisfactory quantitative agreement between the analytical and numerical solutions is achieved when the Ekman number $E$ is sufficiently small. In the nonlinear numerical analysis, we reveal how the excitation and nonlinear interaction of thermal inertial wave modes lead to the weakly turbulent state of inertial convection. We shall concentrate on the moderately supercritical Rayleigh number in the range $1<R a /(R a)_{c}<10$ at a fixed small $E$ and $\operatorname{Pr}$ whose primary dynamics is controlled by the effect of rotation. The paper closes in Sec. V with a brief summary and some remarks.

\section{MATHEMATICAL FORMULATION OF THE PROBLEM}

Consider a Boussinesq fluid confined in an annulus, depicted in Figure 1, with constant thermal diffusivity $\kappa$, constant thermal expansion coefficient $\alpha$, and constant kinematic viscosity $v$. A key geometric assumption for the annulus - whose inner radius $r_{i} d$ and outer radius $r_{o} d$ with the depth $d$ and the aspect ratio denoted by $\Gamma=\left(r_{o} d-r_{i} d\right) / d-$ is that the width of the annulus $\Gamma d=\left(r_{o} d-r_{i} d\right)$ is much smaller than the outer radius $r_{o} d$,

$$
\frac{\Gamma}{r_{o}} \ll 1,
$$

such that we can make a local approximation by neglecting the curvature effect of the annulus. ${ }^{12,20,21}$ This small-gap approximation allows us to adopt simple Cartesian coordinates: azimuthal coordinate $x$, vertical coordinate given by $z$, and inward radial coordinate $y$ with the corresponding unit vectors $(\hat{\mathbf{x}}, \hat{\mathbf{y}}, \hat{\mathbf{z}})$, as displayed in Figure 1 . The similar local approximation has been widely used in the theoretical studies of rotating convection. ${ }^{11}$

The annular channel rotates uniformly with an angular velocity $\mathbf{\Omega}$ in the presence of vertical gravity

$$
\mathbf{g}=-g_{0} \hat{\mathbf{z}},
$$




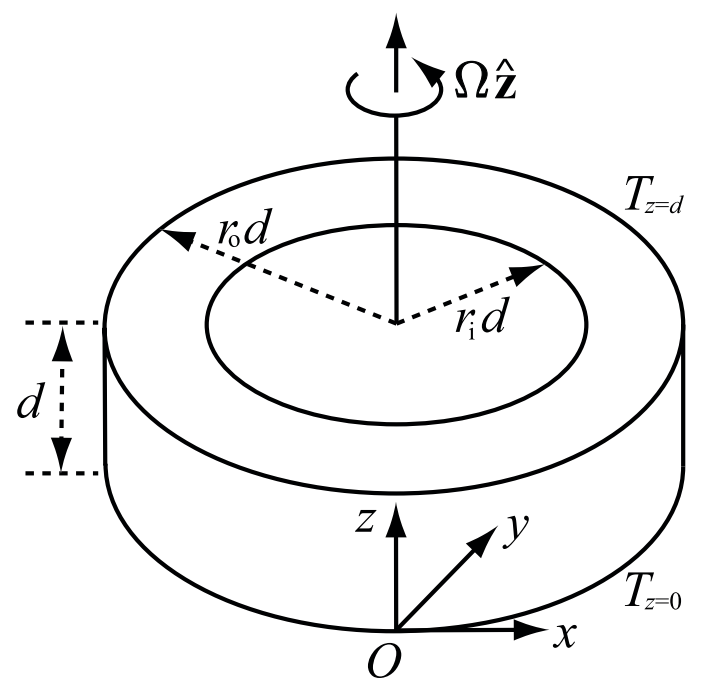

FIG. 1. Geometry of a rotating annulus with inner radius $r_{i} d$ and outer radius $r_{o} d$ rotates uniformly about the symmetry axis with angular velocity $\boldsymbol{\Omega}$. Upon assuming the width of the annulus $\Gamma d=\left(r_{o} d-r_{i} d\right)$ is much smaller than the outer radius $r_{o} d, \Gamma / r_{o} \ll 1$, one can make a local approximation neglecting the curvature effect of the annulus and allow the use of simple Cartesian coordinates: azimuthal coordinate $x$, vertical coordinate given by $z$, and inward radial coordinate $y$.

where $g_{0}$ is constant and $\hat{\mathbf{z}}$ is a unit vector parallel to the angular velocity $\mathbf{\Omega}$. The centrifugal force, which is usually much smaller than the gravity force, is neglected in the present model of rotating convection. Moreover, the annular channel is heated from below to maintain a higher temperature $T_{z=0}$ at the bottom boundary $z=0$ and a lower temperature $T_{z=d}$ at the top boundary $z=d$ with $T_{z=0}>T_{z=d}$. This produces an unstable vertical temperature gradient,

$$
\nabla T_{0}=-\beta \hat{\mathbf{z}},
$$

where $\beta$ is a positive constant, which drives thermal convection when $\beta$ is sufficiently large.

The problem of rotating convection in an annular channel, first formulated and studied by Davies-Jones and Gilman, ${ }^{20}$ and Gilman, ${ }^{12}$ is governed by the three equations

$$
\begin{aligned}
\frac{\partial \mathbf{u}}{\partial t}+\mathbf{u} \cdot \nabla \mathbf{u}+2 \boldsymbol{\Omega} \times \mathbf{u} & =-\frac{1}{\rho} \nabla p+\alpha \Theta g_{0} \hat{\mathbf{z}}+\nu \nabla^{2} \mathbf{u}, \\
\frac{\partial \Theta}{\partial t}+\mathbf{u} \cdot \nabla \Theta & =\beta \mathbf{u} \cdot \hat{\mathbf{z}}+\kappa \nabla^{2} \Theta \\
\nabla \cdot \mathbf{u} & =0
\end{aligned}
$$

where $t$ is the time, $\rho$ is the fluid density, $\Theta$ represents the deviation of the temperature from its static distribution $T_{0}(z), p$ is the total pressure, and $\mathbf{u}$ is the three-dimensional velocity field. We employ the depth $d$ as the length scale, $\Omega^{-1}$ as the unit of time, and $\beta d^{3} \Omega / \kappa$ as the unit of temperature fluctuation of the system, giving rise to the three dimensionless equations

$$
\begin{gathered}
\frac{\partial \mathbf{u}}{\partial t}+\mathbf{u} \cdot \nabla \mathbf{u}+2 \hat{\mathbf{z}} \times \mathbf{u}+\nabla p=\widetilde{R a} \Theta \hat{\mathbf{z}}+E \nabla^{2} \mathbf{u}, \\
\operatorname{Pr}\left(\frac{\partial \Theta}{\partial t}+\mathbf{u} \cdot \nabla \Theta\right)=E\left(\mathbf{u} \cdot \hat{\mathbf{z}}+\nabla^{2} \Theta\right), \\
\nabla \cdot \mathbf{u}=0,
\end{gathered}
$$

where the three non-dimensional parameters, the modified Rayleigh number $\widetilde{R a}$, the Prandtl number $P r$, and the Ekman number $E$ are defined as

$$
\widetilde{R a}=\frac{\alpha \beta g_{0} d^{2}}{\Omega \kappa}, \operatorname{Pr}=\frac{v}{\kappa}, E=\frac{v}{\Omega d^{2}} .
$$


The relation between the usual Rayleigh number $R a$ in non-rotating systems ${ }^{10}$ and the modified Rayleigh number $\widetilde{R a}$ is

$$
R a=\frac{\alpha \beta g_{0} d^{4}}{\nu \kappa}=\frac{\widetilde{R a}}{E} .
$$

The size of $E$, which provides the measure of relative importance between the typical viscous force and the Coriolis force, is assumed to be sufficiently small so that the rotational effect is dynamically predominant. Note that no special symbols are employed to denote the dimensionless variables in (6)-(8) as well as in the rest of the paper.

Equations (6)-(8) are solved subject to the no-slip velocity boundary condition

$$
\hat{\mathbf{n}} \cdot \mathbf{u}=0 \text { and } \hat{\mathbf{n}} \times \mathbf{u}=\mathbf{0}
$$

on the bounding surface $\mathcal{S}$ of the annular channel whose unit normal is denoted by $\hat{\mathbf{n}}$. Two different types of the temperature boundary condition at the sidewalls are adopted. On the top and bottom of a channel, we shall always impose the perfectly conducting condition

$$
\Theta=0 \quad \text { at } \quad z=0,1 .
$$

On the sidewalls, we shall either use the perfectly conducting condition

$$
\Theta=0 \quad \text { at } \quad y=0, \Gamma,
$$

or the perfectly insulating condition

$$
\hat{\mathbf{y}} \cdot \nabla \Theta=0 \quad \text { at } \quad y=0, \Gamma .
$$

The convection problem defined by (6)-(8) subject to an appropriate set of the boundary condition will be solved, first, analytically for the onset of inertial convection at an asymptotically small $E$ in Sec. III and, then, numerically for the onset of inertial convection and its nonlinear developments in Sec. IV.

\section{ASYMPTOTIC SOLUTION FOR INERTIAL CONVECTION}

\section{A. Asymptotic expansion}

In a recent study, Cui et al. ${ }^{25}$ provided the first mathematical proof for the completeness of inertial wave modes in rotating fluid systems by establishing the completeness relation, or Parseval's equality, for any piecewise continuous, differentiable velocity of an incompressible fluid. The completeness of inertial wave modes offers an essential mathematical framework for not only constructing an asymptotic solution for rotating inertial convection at $0<E \ll 1$ but also understanding the nature of its weakly nonlinear development.

Suppose that the mathematical solution of rotating convection in a viscous fluid is piecewise continuous and differentiable. It follows that its velocity $\mathbf{u}$ and pressure $p$ at $0<E \ll 1$ can be always expressed in the form

$$
\begin{aligned}
& \mathbf{u}=\widetilde{\mathbf{u}}+\widehat{\mathbf{u}}+\sum_{m=0}^{M} \sum_{n=0}^{N} \sum_{k=1}^{K}\left[\mathcal{A}_{m n k}(t) \mathbf{u}_{m n k}+c . c\right], \\
& p=\widetilde{p}+\widehat{p}+\sum_{m=0}^{M} \sum_{n=0}^{N} \sum_{k=1}^{K}\left[\mathcal{A}_{m n k}(t) p_{m n k}+\text { c.c. }\right],
\end{aligned}
$$

where $\mathcal{A}_{m n k}$ are complex coefficients with c.c. denoting the complex conjugate of the preceding term, $(\widetilde{\mathbf{u}}, \widetilde{p})$ represents the viscous boundary layer which, by producing a normal mass flux from, or sucking the interior fluid into, the thin viscous boundary layer $\widetilde{\mathbf{u}}$, drives the secondary interior flow $(\widehat{\mathbf{u}}, \widehat{p})$ with $|\widehat{\mathbf{u}}| \ll|\widetilde{\mathbf{u}}|$ and communicates to the interior fluid, and the triple index notation $-m$ is the azimuthal wavenumber while $n$ and $k$ represent the axial and radial wavenumbers of an inertial mode, respectively - is adopted. In expansions (13) and (14), $\mathbf{u}_{m n k}(x, y, z)$ and $p_{m n k}(x, y, z)$ 
represent the spatial part of an inertial mode satisfying

$$
\begin{aligned}
2 \mathrm{i} \sigma_{m n k} \mathbf{u}_{m n k}+2 \hat{\mathbf{z}} \times \mathbf{u}_{m n k}+\nabla p_{m n k} & =0, \\
\nabla \cdot \mathbf{u}_{m n k} & =0,
\end{aligned}
$$

subject to the boundary condition

$$
\hat{\mathbf{n}} \cdot \mathbf{u}_{m n k}=0 \quad \text { on } \quad \mathcal{S},
$$

where $\sigma_{m n k}$ with $\left|\sigma_{m n k}\right|<1$ denotes the half-frequency of an inertial mode. It should be noted that the azimuthal wavenumber $m$, as a result of the small gap approximation, is typically non-integer.

The asymptotic approach based on expansions (13) and (14) has at least four mathematical advantages: (i) an inertial wave mode $\left(\mathbf{u}_{m n k}, p_{m n k}\right)$ has already accommodated the key rotation dynamics of inertial convection in the weakly nonlinear regime, representing the leading-order solution of inertial convection; (ii) the incompressible condition $\nabla \cdot \mathbf{u}=0$ is automatically satisfied; (iii) the boundary condition, $\hat{\mathbf{n}} \cdot \mathbf{u}=0$ on $\mathcal{S}$, is also automatically satisfied with the corresponding viscous boundary layer $\widetilde{\mathbf{u}}$ being readily computable; and (iv) more significantly, the inertial wave mode $\left(\mathbf{u}_{m n k}, p_{m n k}\right)$ is directly associated with the linear differential operator on left-hand side (6) and, thus, an asymptotic solution can be derived at $0<E \ll 1$ if the analytical expression for $\mathbf{u}_{m n k}$ and $p_{m n k}$ is available and simple. It is important to notice that expansions (13) and (14) are profoundly different from the usual Fourier expansion used in a spectral numerical method. This is because expansions (13) and (14) are not only mathematically complete but also, more significantly, their single mode $\left(\mathbf{u}_{m n k}, p_{m n k}\right)$ can represent a leading-order solution of inertial convection.

Our asymptotic analysis for the problem of inertial convection is based on the following scenario. The leading-order interior solution of inertial convection is described by an inviscid inertial wave whose analytical solution is available and mathematically simple. Without having viscous dissipation at leading order, the temperature perturbation $\Theta_{0}$ driven by the inertial wave - which will be referred to as thermal inertial wave - is purely passive, implying that the Rayleigh number $(\widetilde{R a})_{0}=0$ at the leading-order approximation. At next order, a non-zero Rayleigh number, $(\widetilde{R a})_{1} \neq 0$, is required to sustain the thermal inertial wave against the viscous dissipation taking place in both the viscous boundary layer and interior. It seems reasonable, as suggested by the problem of inertial convection in rotating spheres, ${ }^{4,5}$ to anticipate that only a single inertial wave mode $\left(\mathbf{u}_{m n k}, p_{m n k}\right)$ is predominant in expansions (13) and (14) when $0<E \ll 1$ and $0 \leq \operatorname{Pr} \ll 1$. General asymptotic expansions (13) and (14) can be then, for the onset of inertial convection, further simplified to

$$
\begin{aligned}
& \mathbf{u}=\widetilde{\mathbf{u}}+\widehat{\mathbf{u}}+\mathcal{A}_{m n k}(t) \mathbf{u}_{m n k}(x, y, z), \\
& p=\widetilde{p}+\widehat{p}+\mathcal{A}_{m n k}(t) p_{m n k}(x, y, z),
\end{aligned}
$$

along with the expansion

$$
\begin{aligned}
\Theta & =\Theta_{0}+\Theta_{1}+\cdots, \\
\widetilde{R a} & =(\widetilde{R a})_{1}+\cdots, \\
\omega=2 \sigma & =2\left(\sigma_{0}+\sigma_{1}+\cdots\right),
\end{aligned}
$$

where $\left|\mathcal{A}_{m n k} \mathbf{u}_{m n k}\right| \gg|\widehat{\mathbf{u}}|,\left|\mathcal{A}_{m n k} \mathbf{u}_{m n k}\right|=\mathrm{O}(|\widetilde{\mathbf{u}}|), \omega$ is the frequency of inertial convection with $\sigma$ being its half-frequency, and $\sigma_{1}$ represents a small viscous correction to $\sigma_{0}$ with $1>\left|\sigma_{0}\right| \gg$ $\left|\sigma_{1}\right|$. The precise values of the triple wavenumbers $(m, n, k)$ in (17)-(21) and the associated halffrequency $\sigma_{0}$ are to be determined by making use of the condition for the most unstable mode of inertial convection.

It is noteworthy that, in contrast to the classical asymptotic analysis for rotating fluids, ${ }^{26} \sqrt{E}$ is not employed as an expansion parameter in above expansions (17)-(21). This is because the thickness of an oscillatory viscous boundary layer $\widetilde{\mathbf{u}}$ is not generally of order $\sqrt{E}$, the influx from the oscillatory boundary layer is not generally of order $\sqrt{E}$, and the internal viscous contribution is not generally of the order $\sqrt{E}$ smaller than that of the oscillatory boundary layer. ${ }^{27}$ In other words, the effects of spatial and temporal non-uniformities obscure the form of the asymptotic expansion even for the first viscous corrective terms in the problem of oscillatory rotating flows. The only 
requirement for asymptotical expansions (17)-(21) is that the secondary interior flow $\widehat{\mathbf{u}}$ induced by the viscous effect is weak, i.e., $|\widehat{\mathbf{u}}| \ll\left|\mathcal{A}_{m n k} \mathbf{u}_{m n k}\right|$, which is always satisfied when $0<E \ll 1$ for the problem of inertial convection.

\section{B. Non-dissipative thermal inertial wave}

Substitution of expansions (17)-(21) into linearized equations (6)-(8) yields the leading-order interior problem governed by

$$
\begin{aligned}
& 0=\frac{\partial\left(\mathcal{A}_{m n k} \mathbf{u}_{m n k}\right)}{\partial t}+2 \hat{\mathbf{z}} \times\left(\mathcal{A}_{m n k} \mathbf{u}_{m n k}\right)+\nabla\left(\mathcal{A}_{m n k} p_{m n k}\right), \\
& 0=\nabla \cdot\left(\mathcal{A}_{m n k} \mathbf{u}_{m n k}\right), \\
& 0=\operatorname{Pr} \frac{\partial \Theta_{0}}{\partial t}-E\left[\hat{\mathbf{z}} \cdot\left(\mathcal{A}_{m n k} \mathbf{u}_{m n k}\right)+\nabla^{2} \Theta_{0}\right],
\end{aligned}
$$

subject to the inviscid boundary condition

$$
\hat{\mathbf{n}} \cdot \mathbf{u}_{m n k}=0, \text { on } \mathcal{S}
$$

along with a set of temperature boundary conditions (10) and (11) or (10) and (12).

The leading-order interior solution describes a non-dissipative thermal inertial wave in which the momentum equation and the heat equation are decoupled and, consequently, can be solved separately. After performing a straightforward analysis, an analytical solution for the momentum equation reads

$$
\begin{aligned}
\mathcal{A}_{m n k} p_{m n k} & =\left[\frac{\left(k \pi \sigma_{0}\right)}{m \Gamma} \cos \left(\frac{k \pi y}{\Gamma}\right)+\sin \left(\frac{k \pi y}{\Gamma}\right)\right] \cos (n \pi z) \mathrm{e}^{\mathrm{i}\left(m x+2 \sigma_{0} t\right)}, \\
\hat{\mathbf{x}} \cdot\left(\mathcal{A}_{m n k} \mathbf{u}_{m n k}\right) & =\frac{1}{2}\left[\frac{n^{2} \pi^{2}}{m \sigma_{0}} \sin \left(\frac{k \pi y}{\Gamma}\right)-\frac{k \pi}{\Gamma} \cos \left(\frac{k \pi y}{\Gamma}\right)\right] \cos n \pi z \mathrm{e}^{\mathrm{i}\left(m x+2 \sigma_{0} t\right)}, \\
\hat{\mathbf{y}} \cdot\left(\mathcal{A}_{m n k} \mathbf{u}_{m n k}\right) & =\frac{\mathrm{i}}{2}\left[\frac{\left(n^{2} \pi^{2}+m^{2}\right)}{m} \sin \left(\frac{k \pi y}{\Gamma}\right)\right] \cos n \pi z \mathrm{e}^{\mathrm{i}\left(m x+2 \sigma_{0} t\right)}, \\
\hat{\mathbf{z}} \cdot\left(\mathcal{A}_{m n k} \mathbf{u}_{m n k}\right) & =-\frac{\mathrm{i}}{2}\left[\frac{n \pi}{\sigma_{0}} \sin \left(\frac{k \pi y}{\Gamma}\right)+\frac{n k \pi^{2}}{\Gamma m} \cos \left(\frac{k \pi y}{\Gamma}\right)\right] \sin n \pi z \mathrm{e}^{\mathrm{i}\left(m x+2 \sigma_{0} t\right)},
\end{aligned}
$$

where $m>0$ and $n, k$ take positive integer values, and we have taken $\mathcal{A}_{m n k}(t)=\mathrm{e}^{\mathrm{i} 2 \sigma_{0} t}$ with an arbitrary normalization to keep the expression simple and the leading-order half-frequency $\sigma_{0}$ is

$$
\sigma_{0}= \pm \frac{n \pi}{\sqrt{n^{2} \pi^{2}+m^{2}+(k \pi / \Gamma)^{2}}}
$$

satisfying the bound $0<\left|\sigma_{0}\right|<1$. For a given set of the triple wavenumber $(m, n, k)$, there always exist two different thermal inertial waves: a retrograde wave with $\sigma_{0}>0$ and a prograde wave with $\sigma_{0}<0$.

When deriving the leading-order temperature $\Theta_{0}$ for a thermal inertial wave, we have to take into account the type of thermal boundary condition on the sidewalls. For perfectly conducting condition (11), we obtain that

$$
\begin{aligned}
\Theta_{0} & =\sum_{j=1} \frac{\mathrm{i} \pi\left[2 \mathrm{i} \sigma_{0}(\operatorname{Pr} / E)-(j \pi / \Gamma)^{2}-m^{2}-\pi^{2}\right]}{4 \sigma_{0}^{2}(\operatorname{Pr} / E)^{2}+\left[(j \pi / \Gamma)^{2}+m^{2}+\pi^{2}\right]^{2}}\left\{\frac{\delta_{1 j}}{2 \sigma_{0}}+\frac{j\left[1+(-1)^{j}\right]\left(1-\delta_{1 j}\right)}{m \Gamma\left(j^{2}-1\right)}\right\} \\
& \times \sin (\pi z) \sin \left(\frac{j \pi y}{\Gamma}\right) \mathrm{e}^{\mathrm{i}\left(m x+2 \sigma_{0} t\right)},
\end{aligned}
$$

where $\delta_{1 j}=1$ for $j=1$ and $\delta_{1 j}=0$ for $j \neq 1$. In Equation (23), as well as other equations containing the similar term, we set

$$
\frac{\left[1+(-1)^{j}\right]\left(1-\delta_{1 j}\right)}{\left(j^{2}-1\right)}=0 \text { when } j=1
$$


For perfectly insulating condition (12), the temperature $\Theta_{0}$ for a thermal inertial wave is changed to

$$
\begin{aligned}
\Theta_{0} & =\sum_{j=0} \frac{\mathrm{i}\left[2 \mathrm{i} \sigma_{0}(\operatorname{Pr} / E)-(j \pi / \Gamma)^{2}-m^{2}-\pi^{2}\right]}{4 \sigma_{0}^{2}(\operatorname{Pr} / E)^{2}+\left[(j \pi / \Gamma)^{2}+m^{2}+\pi^{2}\right]^{2}}\left\{\left(\frac{\pi^{2}}{2 \Gamma m}\right) \delta_{1 j}+\frac{\left[1+(-1)^{j}\right]\left(1-\delta_{1 j}\right)}{\sigma_{0}\left(1-j^{2}\right)}\right\} \\
& \times \frac{\sin (\pi z)}{S_{k}} \cos \left(\frac{j \pi y}{\Gamma}\right) \mathrm{e}^{\mathrm{i}\left(m x+2 \sigma_{0} t\right)},
\end{aligned}
$$

where $S_{j}=2$ for $j=0, S_{j}=1$ for $j \geq 1$.

The solution of a thermal inertial wave is comprised of an inviscid inertial wave given by $\left(\mathcal{A}_{m n k} \mathbf{u}_{m n k}, \mathcal{A}_{m n k} p_{m n k}\right)$ and the corresponding temperature perturbation $\Theta_{0}$ driven by the inertial wave. Since there are strong inertial effects that play an essential role in breaking the rotational constraint, we anticipate, as confirmed by the asymptotic and numerical analysis, that a non-dissipative thermal inertial wave marked by the simplest vertical $(n=1)$ and radial $(k=1)$ structure would be associated with the most unstable mode of inertial convection for $\Gamma=\mathrm{O}(1)$. This is why we have taken $n=1$ and $k=1$ in (23) and (24) in order to simplify the mathematical expressions. In other words, we shall consider only the inertial wave modes given by $\left(\mathcal{A}_{m 11} \mathbf{u}_{m 11}, \mathcal{A}_{m 11} p_{m 11}\right)$ in (17) and (18) for which the critical wavenumber $m$ will be determined by the associated solvability condition in the next-order problem.

\section{Asymptotic analysis}

It is important to notice that the leading-order interior velocity $\mathcal{A}_{m 11} \mathbf{u}_{m 11}$ in expansion (17) does not satisfy any physical boundary conditions. In a rapidly rotating viscous fluid, no-slip condition (9) would introduce a thin viscous boundary layer $\widetilde{\mathbf{u}}$ such that $\left(\mathcal{A}_{m 11} \mathbf{u}_{m 11}+\widetilde{\mathbf{u}}\right)$ in (17) satisfies the no-slip boundary condition. Physically, it implies that the viscous damping of inertial convection at next order would take place in both the viscous boundary layer and the interior. Mathematically, it implies that an asymptotic matching between the interior perturbation $\widehat{\mathbf{u}}$ and the influx from the boundary-layer solution $\widetilde{\mathbf{u}}$ must be performed to determine the critical parameters of inertial convection.

An explicit solution for the boundary-layer solution $\widetilde{\mathbf{u}}$, which is presented in the Appendix, is required for performing the asymptotic matching in the next-order analysis. With the availability of the boundary layer solution $\widetilde{\mathbf{u}}$, we consider the next-order problem for the secondary interior flow $\widehat{\mathbf{u}}$ governed by

$$
\begin{aligned}
\mathrm{i} 2 \sigma_{0} \widehat{\mathbf{u}}+2 \hat{\mathbf{z}} \times \widehat{\mathbf{u}}+\nabla \widehat{p} & =\widetilde{R a} \Theta_{0} \hat{\mathbf{z}}+E \nabla^{2} \mathbf{u}_{m 11}-\mathrm{i} 2 \sigma_{1} \mathbf{u}_{m 11}, \\
\nabla \cdot \widehat{\mathbf{u}} & =0,
\end{aligned}
$$

subject to the boundary condition on $\mathcal{S}$,

$$
\hat{\mathbf{n}} \cdot \widehat{\mathbf{u}}=\sqrt{E} \int_{0}^{\infty} \hat{\mathbf{n}} \cdot \nabla \times(\hat{\mathbf{n}} \times \widetilde{\mathbf{u}}) \mathrm{d} \xi
$$

where $\xi=y / \sqrt{E}$ denotes the boundary-layer coordinate on the outer sidewall, $\xi=(\Gamma-y) / \sqrt{E}$ on the inner sidewall, $\xi=z / \sqrt{E}$ on the bottom, and $\xi=(1-z) / \sqrt{E}$ on the top. The critical azimuthal wavenumber $m_{c}$, the critical frequency $\omega_{c}$, and the critical Rayleigh number $(\widetilde{R a})_{c}$ are determined by the solvability condition required for inhomogeneous systems (25) and (26), which is

$$
\begin{aligned}
-2 \sqrt{E}\{ & \int_{0}^{\Gamma}\left(\mathrm{i} \sigma_{0} \mathbf{u}_{m 11}^{*}-\hat{\mathbf{z}} \times \mathbf{u}_{m 11}^{*}\right)_{z=0} \cdot\left(\int_{0}^{\infty} \widetilde{\mathbf{u}}_{\text {bottom }} \mathrm{d} \xi\right) \mathrm{d} y \\
& +\int_{0}^{\Gamma}\left(\mathrm{i} \sigma_{0} \mathbf{u}_{m 11}^{*}-\hat{\mathbf{z}} \times \mathbf{u}_{m 11}^{*}\right)_{z=1} \cdot\left(\int_{0}^{\infty} \widetilde{\mathbf{u}}_{\text {top }} \mathrm{d} \xi\right) \mathrm{d} y \\
& +\int_{0}^{1}\left(\mathrm{i} \sigma_{0} \mathbf{u}_{m 11}^{*}-\hat{\mathbf{z}} \times \mathbf{u}_{m 11}^{*}\right)_{y=0} \cdot\left(\int_{0}^{\infty} \widetilde{\mathbf{u}}_{\text {outer }} \mathrm{d} \xi\right) \mathrm{d} z \\
& \left.+\int_{0}^{1}\left(\mathrm{i} \sigma_{0} \mathbf{u}_{m 11}^{*}-\hat{\mathbf{z}} \times \mathbf{u}_{m 11}^{*}\right)_{y=1} \cdot\left(\int_{0}^{\infty} \widetilde{\mathbf{u}}_{\text {inner }} \mathrm{d} \xi\right) \mathrm{d} z\right\}
\end{aligned}
$$




$$
\begin{aligned}
& =\left[\widetilde{R a} \int_{0}^{1} \int_{0}^{\Gamma} \mathbf{u}_{m 11}^{*} \cdot \hat{\mathbf{z}} \Theta_{0} \mathrm{~d} y \mathrm{~d} z+E \int_{0}^{1} \int_{0}^{\Gamma} \mathbf{u}_{m 11}^{*} \cdot \nabla^{2} \mathbf{u}_{m 11} \mathrm{~d} y \mathrm{~d} z\right. \\
& \left.-\mathrm{i} 2 \sigma_{1} \int_{0}^{1} \int_{0}^{\Gamma}\left|\mathbf{u}_{m 11}\right|^{2} \mathrm{~d} y \mathrm{~d} z\right],
\end{aligned}
$$

where $\mathbf{u}_{m 11}^{*}$ denotes the complex conjugate of $\mathbf{u}_{m 11}$ and four integrals on the left side represent the influx from the four boundary layers with the corner effects being neglected. After carrying out all the integrations in solvability condition (27), we obtain a complex equation whose real part determines the critical wavenumber $m_{c}$ and the critical Rayleigh number $(\widetilde{R a})_{c}$ while whose imaginary part gives rise to the viscous correction $\sigma_{1}$.

Consider two different types of the temperature condition at the sidewalls. In the case of the perfectly conducting sidewalls, we obtain, after making use of boundary-layer solutions (A4)-(A7) and the temperature $\Theta_{0}$ given by (23), an expression for the marginal Rayleigh number

$$
\begin{aligned}
& \widetilde{R a}=\left\{\frac{E \pi^{2} \Gamma^{2}}{4 \sigma_{0}^{2}}\left[\left(1+\frac{\pi^{2}}{m^{2}}\right)\left(\frac{1}{\sigma_{0}^{2}}+\frac{1}{\Gamma^{2}}\right)+\frac{\left(\pi^{2}+m^{2}\right)^{2}}{\pi^{2} m^{2}}\right]+\mathcal{I}_{1} \sqrt{E}\right\} \\
& \left\{\sum_{j=1}^{J} \frac{\pi^{2}+m^{2}+(j \pi / \Gamma)^{2}}{\left[\pi^{2}+m^{2}+(j \pi / \Gamma)^{2}\right]^{2}+\left(2 \sigma_{0} \operatorname{Pr} / E\right)^{2}}\left[\frac{\Gamma \delta_{1 j}}{2 \sigma_{0}}+\frac{j\left[1+(-1)^{j}\right]\left(1-\delta_{1 j}\right)}{m\left(j^{2}-1\right)}\right]^{2}\right\}^{-1}
\end{aligned}
$$

and the corresponding frequency of inertial convection

$$
\begin{aligned}
& \omega=2 \sigma_{0}-\left\{I_{2} \sqrt{E}+\sum_{j=1}^{J} \frac{8 \sigma_{0} \widetilde{R a} \operatorname{Pr} / E}{\left[\pi^{2}+m^{2}+(j \pi / \Gamma)^{2}\right]^{2}+\left(2 \sigma_{0} \operatorname{Pr} / E\right)^{2}}\left(\frac{1}{\Gamma^{2}}\right)\right. \\
& \left.\left[\frac{\Gamma \delta_{1 j}}{2 \sigma_{0}}+\left(\frac{j\left[1+(-1)^{j}\right]\left(1-\delta_{1 j}\right)}{m\left(j^{2}-1\right)}\right)\right]^{2}\right\}\left[\left(1+\frac{\pi^{2}}{m^{2}}\right)\left(\frac{1}{\sigma_{0}^{2}}+\frac{1}{\Gamma^{2}}\right)+\frac{\left(\pi^{2}+m^{2}\right)^{2}}{\pi^{2} m^{2}}\right]^{-1},
\end{aligned}
$$

where $\mathcal{I}_{1}$ and $\mathcal{I}_{2}$ are

$$
\begin{aligned}
\mathcal{I}_{1} & =\Gamma^{2} \sqrt{1+\sigma_{0}}\left\{\frac{1}{2}\left[\frac{\pi^{2}}{m^{2} \sigma_{0}^{2}}+\frac{\left(\pi^{2}+m^{2}\right)^{2}}{\pi^{2} m^{2}}+\frac{1}{\Gamma^{2}}\right]-\frac{\left(\pi^{2}+m^{2}\right)}{m^{2} \sigma_{0}}\right\} \\
& +\Gamma^{2} \sqrt{1-\sigma_{0}}\left\{\frac{1}{2}\left[\frac{\pi^{2}}{m^{2} \sigma_{0}^{2}}+\frac{\left(\pi^{2}+m^{2}\right)^{2}}{\pi^{2} m^{2}}+\frac{1}{\Gamma^{2}}\right]+\frac{\left(\pi^{2}+m^{2}\right)}{m^{2} \sigma_{0}}\right\}+\frac{\sqrt{\left|\sigma_{0}\right|}}{\Gamma}\left(1+\frac{\pi^{2}}{m^{2}}\right), \\
\mathcal{I}_{2} & =\sqrt{1+\sigma_{0}}\left\{2\left[\frac{\pi^{2}}{m^{2} \sigma_{0}^{2}}+\frac{\left(\pi^{2}+m^{2}\right)^{2}}{\pi^{2} m^{2}}+\frac{1}{\Gamma^{2}}\right]-\frac{4\left(\pi^{2}+m^{2}\right)}{m^{2} \sigma_{0}}\right\} \\
& -\sqrt{1-\sigma_{0}}\left\{2\left[\frac{\pi^{2}}{m^{2} \sigma_{0}^{2}}+\frac{\left(\pi^{2}+m^{2}\right)^{2}}{\pi^{2} m^{2}}+\frac{1}{\Gamma^{2}}\right]+\frac{4\left(\pi^{2}+m^{2}\right)}{m^{2} \sigma_{0}}\right\}+\frac{4 \sigma_{0}}{\Gamma^{3} \sqrt{\left|\sigma_{0}\right|}}\left(1+\frac{\pi^{2}}{m^{2}}\right) .
\end{aligned}
$$

In (28) and (29), $J=\mathrm{O}(10)$ would be sufficient for achieving $1 \%$ accuracy. Since $\sigma_{0}$ given by (22) is only a function of $m$ with $n=1$ and $k=1$, expression (28) for $\widetilde{R a}$ is also only a function of $m$ and can be readily minimized with respect to the wavenumber $m$, giving rise to the critical wavenumber $m_{c}$ and the critical Rayleigh number $\left.\widetilde{R a}\right)_{c}$. Inserting $m=m_{c}$ and $\widetilde{R a}=(\widetilde{R a})_{c}$ into (29), we can then compute the viscous correction $2 \sigma_{1}$ for the critical frequency $\omega_{c}$ of inertial convection. It is important to note the symmetry

$$
\mathcal{I}_{1}\left(\sigma_{0}\right)=\mathcal{I}_{1}\left(-\sigma_{0}\right) \widetilde{R a}\left(\sigma_{0}\right)=\widetilde{R a}\left(-\sigma_{0}\right),
$$

which implies that there always exist two different solutions of inertial convection - the retrograde thermal inertial wave with $\sigma_{0}>0$ denoted by $\left(\mathbf{u}^{+}, \Theta_{0}^{+}\right)$and the prograde thermal inertial wave with $\sigma_{0}<0$ denoted by $\left(\mathbf{u}^{-}, \Theta_{0}^{-}\right)$- that have exactly the same critical Rayleigh number $(\widetilde{R a})_{c}$ but different spatial structures $\mathbf{u}^{+} \neq \mathbf{u}^{-}$. Note that there would exist small differences, such as the critical Rayleigh number, between two counter-traveling inertial waves in a narrow annulus without making the small-gap approximation. This feature is especially highlighted because of its significance in understanding the properties of nonlinear inertial convection. 
It is fortunate that the asymptotic solution of the convective flow $\mathbf{u}^{ \pm}$can be written in closed form for any given parameters $E, P r$, and $\Gamma$ with the no-slip boundary condition. After obtaining the critical wavenumber $m_{c}$ by minimizing (28) with respect to $m$, the velocity $\mathbf{u}^{ \pm}$of inertial convection can be expressible as

$$
\begin{aligned}
\hat{\mathbf{x}} \cdot \mathbf{u}^{ \pm} & =\left\{\frac{\cos \pi z}{2}\left[\frac{ \pm \pi \sqrt{\pi^{2}\left(1+1 / \Gamma^{2}\right)+m_{c}^{2}}}{m_{c}} \sin \frac{\pi y}{\Gamma}-\frac{\pi}{\Gamma}\left(\cos \frac{\pi y}{\Gamma}+\mathrm{e}^{-\gamma_{3}(\Gamma-y)}-\mathrm{e}^{-\gamma_{3} y}\right)\right]\right. \\
& +\frac{\left(\pi^{2}+m_{c}^{2}\right)}{4 m_{c}} \sin \frac{\pi y}{\Gamma}\left[\mathrm{e}^{-\gamma_{1} z}-\mathrm{e}^{-\gamma_{2} z}-\mathrm{e}^{-\gamma_{1}(1-z)}+\mathrm{e}^{-\gamma_{2}(1-z)}\right] \\
& +\frac{\pi}{4 \Gamma}\left[\left(\mathrm{e}^{-\gamma_{1} z}+\mathrm{e}^{-\gamma_{2} z}-\mathrm{e}^{-\gamma_{1}(1-z)}-\mathrm{e}^{-\gamma_{2}(1-z)}\right)\right. \\
& \left.\left.\times\left(\cos \frac{\pi y}{\Gamma}-\frac{ \pm \Gamma \sqrt{\pi^{2}\left(1+1 / \Gamma^{2}\right)+m_{c}^{2}}}{m_{c}} \sin \frac{\pi y}{\Gamma}\right)\right]\right\} \mathrm{e}^{\mathrm{i}\left\{m_{c} x \pm 2 \pi t / \sqrt{\pi^{2}\left(1+1 / \Gamma^{2}\right)+m_{c}^{2}}\right\}}, \\
\hat{\mathbf{y}} \cdot \mathbf{u}^{ \pm} & =\left\{\frac { \mathrm { i } ( \pi ^ { 2 } + m _ { c } ^ { 2 } ) } { 4 m _ { c } } \operatorname { s i n } \frac { \pi y } { \Gamma } \left[\left(2 \cos \pi z-\mathrm{e}^{-\gamma_{1} z}-\mathrm{e}^{-\gamma_{2} z}+\mathrm{e}^{-\gamma_{1}(1-z)}+\mathrm{e}^{-\gamma_{2}(1-z)}\right)\right.\right. \\
& +\frac{\mathrm{i} \pi}{4 \Gamma}\left(\cos \frac{\pi y}{\Gamma}-\frac{ \pm \Gamma \sqrt{\pi^{2}\left(1+1 / \Gamma^{2}\right)+m_{c}^{2}}}{m_{c}} \sin \frac{\pi y}{\Gamma}\right)\left[-\mathrm{e}^{-\gamma_{1} z}+\mathrm{e}^{-\gamma_{2} z}\right. \\
& \left.\left.\left.+\mathrm{e}^{-\gamma_{1}(1-z)}-\mathrm{e}^{-\gamma_{2}(1-z)}\right]\right\} \mathrm{e}^{\mathrm{i}\left\{m_{c} x \pm 2 \pi t / \sqrt{\pi^{2}\left(1+1 / \Gamma^{2}\right)+m_{c}^{2}}\right.}\right\} \\
\hat{\mathbf{z}} \cdot \mathbf{u}^{ \pm} & =-\frac{\mathrm{i}}{2}\left[ \pm \sqrt{\pi^{2}\left(1+1 / \Gamma^{2}\right)+m_{c}^{2}} \sin \left(\frac{\pi y}{\Gamma}\right)+\frac{\pi^{2}}{\Gamma m_{c}}\left(\cos \frac{\pi y}{\Gamma}-\mathrm{e}^{-\gamma_{3}(\Gamma-y)}+\mathrm{e}^{-\gamma_{3} y}\right)\right] \\
& \times \sin \pi z \mathrm{e}^{\mathrm{i}\left\{m_{c} x \pm 2 \pi t / \sqrt{\pi^{2}\left(1+1 / \Gamma^{2}\right)+m_{c}^{2}}\right\}},
\end{aligned}
$$

where

$$
\begin{aligned}
& \gamma_{1}=\frac{(1+\mathrm{i})}{\sqrt{E}}\left(1+\frac{ \pm \pi}{\sqrt{\pi^{2}\left(1+1 / \Gamma^{2}\right)+m_{c}^{2}}}\right)^{1 / 2}, \\
& \gamma_{2}=\frac{(1-\mathrm{i})}{\sqrt{E}}\left(1-\frac{ \pm \pi}{\sqrt{\pi^{2}\left(1+1 / \Gamma^{2}\right)+m_{c}^{2}}}\right)^{1 / 2}, \\
& \gamma_{3}=\frac{(1 \pm \mathrm{i})}{\sqrt{E}}\left(0+\frac{\pi}{\sqrt{\pi^{2}\left(1+1 / \Gamma^{2}\right)+m_{c}^{2}}}\right)^{1 / 2} .
\end{aligned}
$$

The asymptotic solution for the corresponding temperature $\Theta_{0}^{ \pm}$can be obtained by letting $m=m_{c}$ and $\sigma_{0}= \pm \pi / \sqrt{\pi^{2}\left(1+1 / \Gamma^{2}\right)+m_{c}^{2}}$ in (23).

In the case of the two insulating sidewalls, we can also derive the expressions for the Rayleigh number $\widetilde{R a}$ and the frequency $\omega$ from solvability condition (27) using boundary-layer solutions (A4)-(A7) but with the temperature $\Theta_{0}$ given by (24), which are

$$
\begin{aligned}
& \widetilde{R a}=\left\{\frac{E \pi^{4} \Gamma^{2}}{4 \sigma_{0}^{2}}\left[\left(1+\frac{\pi^{2}}{m^{2}}\right)\left(\frac{1}{\sigma_{0}^{2}}+\frac{1}{\Gamma^{2}}\right)+\frac{\left(\pi^{2}+m^{2}\right)^{2}}{\pi^{2} m^{2}}\right]+\mathcal{I}_{1} \pi^{2} \sqrt{E}\right\} \\
& \left\{\sum_{j=0}^{J} \frac{\pi^{2}+m^{2}+(j \pi / \Gamma)^{2}}{\left[\pi^{2}+m^{2}+(j \pi / \Gamma)^{2}\right]^{2}+\left(2 \sigma_{0} P r / E\right)^{2}} \frac{1}{S_{j}}\left[\frac{\pi^{2} \delta_{1 j}}{2 m}+\frac{\Gamma\left[1+(-1)^{j}\right]\left(1-\delta_{1 j}\right)}{\sigma_{0}\left(1-j^{2}\right)}\right]^{2}\right\}^{-1}
\end{aligned}
$$

and

$$
\begin{gathered}
\omega=2 \sigma_{0}-\left\{I_{2} \sqrt{E}+\sum_{j=0}^{J} \frac{8 \sigma_{0} \widetilde{R a P r} / E}{\left[\pi^{2}+m^{2}+(j \pi / \Gamma)^{2}\right]^{2}+\left(2 \sigma_{0} \operatorname{Pr} / E\right)^{2}}\left(\frac{1}{S_{j} \Gamma^{2} \pi^{2}}\right)\right. \\
\left.\left[\frac{\pi^{2} \delta_{1 j}}{2 m}+\frac{\Gamma\left[1+(-1)^{j}\right]\left(1-\delta_{1 j}\right)}{\sigma_{0}\left(1-j^{2}\right)}\right]^{2}\right\}\left[\left(1+\frac{\pi^{2}}{m^{2}}\right)\left(\frac{1}{\sigma_{0}^{2}}+\frac{1}{\Gamma^{2}}\right)+\frac{\left(\pi^{2}+m^{2}\right)^{2}}{\pi^{2} m^{2}}\right]^{-1} .
\end{gathered}
$$


TABLE I. Several typical values of the critical Rayleigh number $(\widetilde{R a})_{c}$, the critical azimuthal wavenumber $m_{c}$, and the critical half-frequency $\sigma_{c}$ at the onset of retrograde convection $\left(\sigma_{c}>0\right)$ for $\operatorname{Pr}=0.023$ and $\Gamma=1.0$ with the no-slip velocity condition and the perfectly conducting temperature condition on $\mathcal{S}$. The result of the fully numerical solution is indicated by the subscript num while the asymptotic solution, indicated by the subscript asym, is computed from analytical expressions (28) and (29).

\begin{tabular}{lcc}
\hline \hline$E$ & {$\left[(\widetilde{R a})_{c}, m_{c}, \sigma_{c}\right]_{\text {num }}$} & {$\left[(\widetilde{R a})_{c}, m_{c}, \sigma_{c}\right]_{\text {asym }}$} \\
\hline $10^{-2}$ & $(68.220,2.8834,0.4692)$ & $(55.296,2.7605,0.6196)$ \\
$5.0 \times 10^{-3}$ & $(41.448,2.9584,0.5315)$ & $(33.770,2.9176,0.5943)$ \\
$10^{-3}$ & $(18.896,4.4048,0.4738)$ & $(16.443,4.3801,0.4865)$ \\
$5.0 \times 10^{-4}$ & $(17.391,5.8688,0.4035)$ & $(15.564,5.8612,0.4102)$ \\
$10^{-4}$ & $(19.613,10.885,0.2536)$ & $(18.333,10.936,0.2545)$ \\
$5.0 \times 10^{-5}$ & $(21.942,13.892,0.2047)$ & $(20.830,13.978,0.2048)$ \\
$10^{-5}$ & $(30.759,23.953,0.1230)$ & $(29.633,24.128,0.1226)$ \\
\hline \hline
\end{tabular}

Expression (33) for $\widetilde{R a}$ can be also easily minimized with respect to the wavenumber $m$ to provide the critical wavenumber $m_{c}$ and the critical Rayleigh number $(\widetilde{R a})_{c}$. In parallel to the conducting sidewalls, there also exist two different solutions - the retrograde thermal inertial wave with $\sigma_{0}>0$ and the prograde thermal inertial wave with $\sigma_{0}<0$ - which have exactly the same critical Rayleigh number $(\widetilde{R a})_{c}$ whose velocity $\mathbf{u}^{ \pm}$is also given by (30)-(32) but its temperature $\Theta_{0}^{ \pm}$is obtained by letting $m=m_{c}$ and $\sigma_{0}= \pm \pi / \sqrt{\pi^{2}\left(1+1 / \Gamma^{2}\right)+m_{c}^{2}}$ in (24).

Several typical values of the critical parameters, $(\widetilde{R a})_{c}, m_{c}$, and $\sigma_{c}$, for the onset of inertial convection with $\operatorname{Pr}=0.023$ (the fluid metal gallium) and $\Gamma=1.0$ for the retrograde convection $\sigma_{c}>0$, computed from analytical expressions (28) and (29) for the perfectly conducting sidewalls, are presented in Table I for different values of the Ekman number $E$ along with the corresponding numerical results. Similarly, several typical solutions for the insulating sidewalls calculated from analytical expressions (33) and (34) are shown in Table II. For the insulating sidewalls, the spatial structure of prograde inertial convection from the asymptotic solution $\mathbf{u}^{+}$with $\operatorname{Pr}=0.023$ and $\Gamma=1$ is shown for $E=10^{-3}$ in Figures 2(a)-2(c) and for $E=10^{-4}$ in Figures 3(a)-3(c). It should be noted that all the cases presented in Tables I and II represent the most unstable mode of convection. Detailed discussion about the asymptotic solution and its comparison with the fully numerical solution will be presented in Sec. IV.

TABLE II. Several typical values of the critical Rayleigh number $(\widetilde{R a})_{c}$, the critical azimuthal wavenumber $m_{c}$, and the critical half-frequency $\sigma_{c}$ at the onset of inertial convection for $\operatorname{Pr}=0.023$ and $\Gamma=1.0$ in a rotating channel with the no-slip velocity boundary condition on $\mathcal{S}$, the perfectly conducting temperature condition at the top and bottom, and the insulating temperature condition at the two sidewalls. The result of the fully numerical solution is indicated by a subscript num while the asymptotic solution denoted by a subscript asym is computed from analytical expressions (33) and (34).

\begin{tabular}{lcc}
\hline \hline$E$ & {$\left[(\widetilde{R a})_{c}, m_{c}, \sigma_{c}\right]_{\text {num }}$} & {$\left[(\widetilde{R a})_{c}, m_{c}, \sigma_{c}\right]_{\text {asym }}$} \\
\hline $10^{-2}$ & $(43.241,2.2221,0.5302)$ & $(32.543,1.7174,0.6544)$ \\
$5.0 \times 10^{-3}$ & $(28.735,2.1481,0.5627)$ & $(21.620,1.9925,0.6234)$ \\
$10^{-3}$ & $(18.284,4.5298,0.4595)$ & $(15.205,4.4985,0.4732)$ \\
$5.0 \times 10^{-4}$ & $(17.413,6.0505,0.3930)$ & $(15.310,6.0685,0.3986)$ \\
$10^{-4}$ & $(19.696,10.936,0.2518)$ & $(18.403,11.040,0.2515)$ \\
$5.0 \times 10^{-5}$ & $(21.992,13.942,0.2040)$ & $(20.888,14.046,0.2038)$ \\
$10^{-5}$ & $(30.777,23.970,0.1229)$ & $(29.659,24.155,0.1279)$ \\
\hline \hline
\end{tabular}



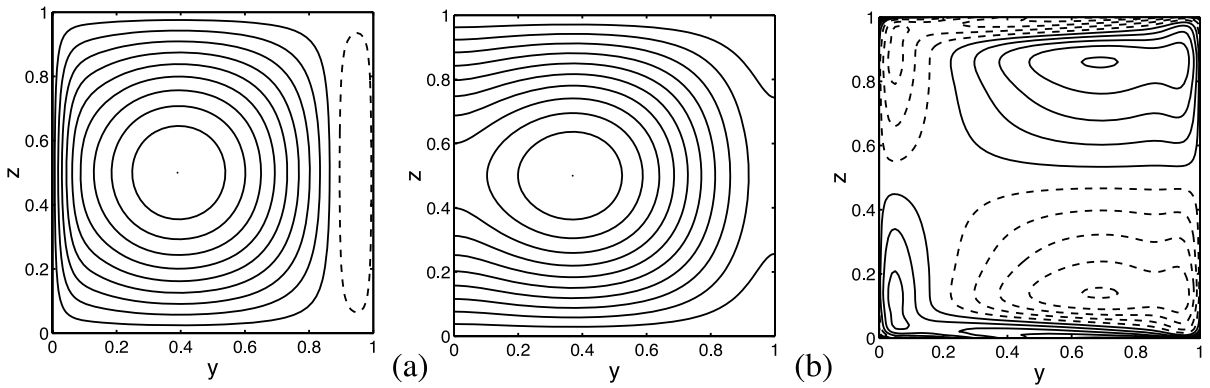

(c)
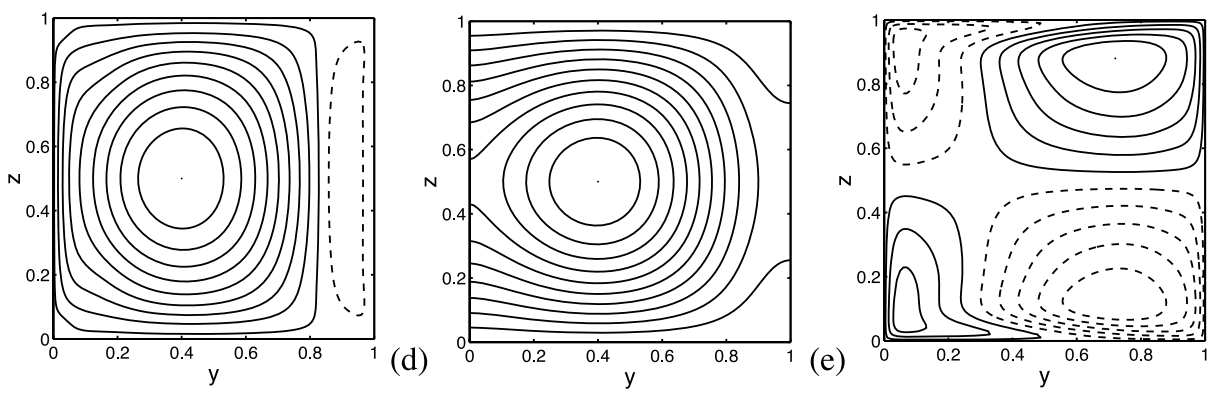

FIG. 2. Displayed in a vertical y-z plane are contours of (a) $\hat{\mathbf{z}} \cdot \mathbf{u},(\mathrm{b}) \Theta$, and (c) $\hat{\mathbf{x}} \cdot \mathbf{u}$ calculated using the analytical solution with $E=10^{-3}, P r=0.023, \Gamma=1$ in a rotating annular channel with the conducting top and bottom and the two insulating sidewalls. The corresponding numerical solution for the same parameters is presented in (d)-(f).

\section{NUMERICAL ANALYSIS}

\section{A. Onset of inertial convection}

The primary objective of the linear numerical analysis, which is valid for large and moderately small values of the Ekman number $E$, is to provide a valuable comparison with the result of the asymptotic analysis that is valid only for $0<E \ll 1$. In contrast to the problem of the classical
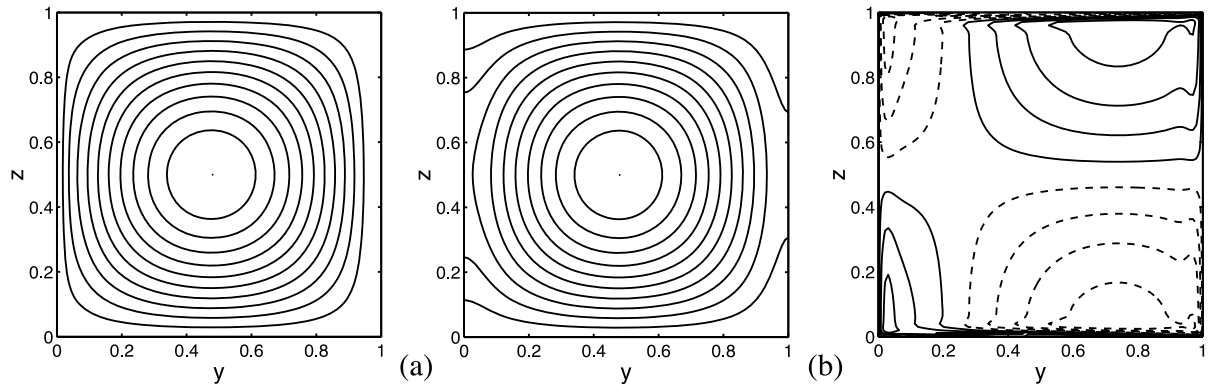

(c)
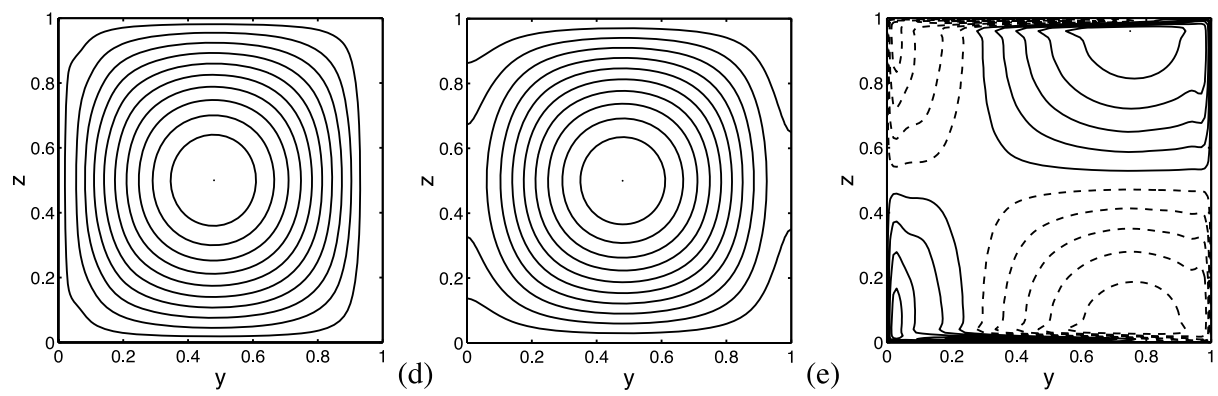

FIG. 3. Displayed in a vertical $y-z$ plane are contours of (a) $\hat{\mathbf{z}} \cdot \mathbf{u}$, (b) $\Theta$, and (c) $\hat{\mathbf{x}} \cdot \mathbf{u}$ calculated from the analytical solution with $E=10^{-4}, \operatorname{Pr}=0.023, \Gamma=1$ with the conducting top and bottom and the two insulating sidewalls. The corresponding numerical solution is shown in (d)-(f). 
Rayleigh-Bénard problem, the non-slip boundary condition at the two sidewalls dramatically increases the complexity of the numerical analysis. We express the velocity $\mathbf{u}$ of inertial convection in terms of two scalar potentials $\Psi$ and $\Phi$,

$$
\mathbf{u}=\nabla \times[\Psi(x, y, z, t) \hat{\mathbf{y}}]+\nabla \times[\Phi(x, y, z, t) \hat{\mathbf{z}}] .
$$

Making use of this expression and applying $\hat{\mathbf{y}} \cdot \nabla \times$ and $\hat{\mathbf{z}} \cdot \nabla \times$ onto linearized momentum equation (6), we derive the three scalar partial differential equations that govern the onset of inertial convection

$$
\begin{aligned}
& 0=\left(\frac{\partial}{\partial t}-E \nabla^{2}\right)\left[-\left(\frac{\partial^{2}}{\partial x^{2}}+\frac{\partial^{2}}{\partial z^{2}}\right) \Psi+\frac{\partial^{2} \Phi}{\partial y \partial z}\right]+2 \frac{\partial^{2} \Phi}{\partial x \partial z}+\widetilde{R a} \frac{\partial \Theta}{\partial x}, \\
& 0=\left(\frac{\partial}{\partial t}-E \nabla^{2}\right)\left[-\left(\frac{\partial^{2}}{\partial x^{2}}+\frac{\partial^{2}}{\partial y^{2}}\right) \Phi+\frac{\partial^{2} \Psi}{\partial y \partial z}\right]-2 \frac{\partial^{2} \Psi}{\partial x \partial z}, \\
& 0=\left(\operatorname{Pr} \frac{\partial}{\partial t}-E \nabla^{2}\right) \Theta-E \frac{\partial \Psi}{\partial x} .
\end{aligned}
$$

In terms of $\Psi$ and $\Phi$, the no-slip condition on the top and bottom imposes

$$
\Psi=\frac{\partial \Psi}{\partial z}=\Phi=0 \quad \text { at } z=0,1,
$$

while the two no-slip sidewalls require

$$
\Psi=\Phi=\frac{\partial \Phi}{\partial y}=0 \quad \text { at } y=0, \Gamma .
$$

Upon further writing $\Psi$ and $\Phi$, along with the temperature $\Theta$, in the form

$$
\begin{aligned}
& \Psi(x, y, z, t)=\Psi(y, z) \mathrm{e}^{\mathrm{i}(m x+\omega t)}, \\
& \Phi(x, y, z, t)=\Phi(y, z) \mathrm{e}^{\mathrm{i}(m x+\omega t)}, \\
& \Theta(x, y, z, t)=\Theta(y, z) \mathrm{e}^{\mathrm{i}(m x+\omega t)},
\end{aligned}
$$

we can solve Equations (36)-(38) numerically by virtue of the Galerkin-type spectral expansion for $\Psi(y, z)$ and $\Phi(y, z)$,

$$
\begin{aligned}
& \Psi(y, z)=\sum_{l=0}^{N} \sum_{k=0}^{N} \Psi_{k l}\left[\left(1-\hat{z}^{2}\right)^{2} T_{l}(\hat{z})\right]\left[\left(1-\hat{y}^{2}\right) T_{k}(\hat{y})\right], \\
& \Phi(y, z)=\sum_{l=0}^{N} \sum_{k=0}^{N} \Phi_{k l}\left[\left(1-\hat{z}^{2}\right) T_{l}(\hat{z})\right]\left[\left(1-\hat{y}^{2}\right)^{2} T_{k}(\hat{y})\right],
\end{aligned}
$$

where $\hat{y}=(2 y / \Gamma-1), \hat{z}=(2 z-1), \Psi_{k l}$ and $\Phi_{k l}$ are complex coefficients, $N$ denotes the truncation parameter taken to be about 100 sufficiently for achieving $1 \%$ accuracy for the parameters considered in this paper, and $T_{l}(x)$ represents the standard Chebyshev functions. For the conducting sidewalls, we expand the temperature $\Theta$ as

$$
\Theta(y, z)=\sum_{l=0}^{N} \sum_{k=1}^{N} \Theta_{k l}\left[\left(1-\hat{z}^{2}\right) T_{l}(\hat{z})\right]\left[\sin \frac{k \pi}{2}(\hat{y}+1)\right],
$$

while for the insulating sidewalls, we take

$$
\Theta(y, z)=\sum_{l=0}^{N} \sum_{k=0}^{N} \widehat{\Theta}_{k l}\left[\left(1-\hat{z}^{2}\right) T_{l}(\hat{z})\right]\left[\cos \frac{k \pi}{2}(\hat{y}+1)\right] .
$$

Substituting the above expansions into (36)-(38) and applying a standard numerical procedure yield a system of the nonlinear algebraic equations for which the coefficients $\Psi_{k l}, \Phi_{k l}, \Theta_{k l}$ (or $\widehat{\Theta}_{k l}$ ), the Rayleigh number $\widetilde{R a}$, and the frequency $\omega$ are unknown. The system is then solved by an iterative scheme that determines the critical parameters $\left[(\widetilde{R a})_{c}, m_{c}, \omega_{c}\right]$ for the most unstable mode of convection as well as all the coefficients $\Psi_{k l}, \Phi_{k l}, \Theta_{k l}$ (or $\widehat{\Theta}_{k l}$ ). In the iterative process, coefficients 
$\Psi_{k l}, \Phi_{k l}, \Theta_{k l}$ (or $\widehat{\Theta}_{k l}$ ) with a proper normalization, the Rayleigh number $\widetilde{R a}$, and the frequency $\omega$ are first obtained by solving the nonlinear algebraic equations for a given wavenumber $m$, and then, through a further iteration over $m$, the critical parameters $\left[(\widetilde{R a})_{c}, m_{c}, \omega_{c}\right]$ are determined.

Several values of the critical Rayleigh number $(\widetilde{R a})_{c}$, the critical azimuthal wavenumber $m_{c}$, and the critical half-frequency $\sigma_{c}$ computed from the numerical analysis are presented in Table I for the conducting sidewalls and Table II for the insulating sidewalls with $\operatorname{Pr}=0.023$ and $\Gamma=1.0$ at different values of $E$. A satisfactory agreement between the asymptotic and numerical solutions is achieved for sufficiently small $E$ in both cases. An important question is how small $E$ is required in order that the asymptotic solution valid only for $0<E \ll 1$ offers a reasonable approximation. For $E=10^{-2}$, both Tables I and II show that there exist substantial differences between the asymptotic and numerical values, suggesting that the dynamics of inertial convection for $E \geq 10^{-2}$ is not primarily controlled by the effect of rotation. For $E \leq 10^{-3}$, Tables I and II show a quantitative agreement between the asymptotic and numerical solutions expected from the leading-order solution in asymptotic expansions (20) and (21). The spatial structures of inertial convection computed from the analytical solution and the corresponding numerical solution at exactly the same parameter are depicted in Figure 2 for the case of two insulating sidewalls at $E=10^{-3}$, showing insignificant differences between the numerical and analytical solutions. For a smaller Ekman number $E=10^{-4}$, while the numerical analysis for the insulating sidewalls gives rise to $m_{c}=10.94, \sigma_{c}=0.252,(\widetilde{R a})_{c}=19.70$, asymptotic formulas (33) and (34) yield $m_{c}=11.04, \sigma_{c}=0.252,(\widetilde{R a})_{c}=18.40$. Moreover, there exist no noticeable differences between the numerical and analytical solutions at $E=10^{-4}$, which are displayed in Figure 3. It suggests that the dynamics of inertial convection for the liquid metal gallium with $\mathrm{Pr}=0.023$ is primarily controlled by the effect of rotation when $E \leq 10^{-3}$.

Another interesting feature is that the difference between the conducting and insulating sidewalls, comparing Table I to Table II, diminishes when $E$ becomes sufficiently small, highlighting that the nature of thermal condition on the sidewalls is of secondary significance for sufficiently small $E$. For instance, the analytical solution at $E=10^{-5}$ for the insulating sidewalls gives $m_{c}=24.16, \sigma_{c}=0.128,(\widetilde{R a})_{c}=29.66$ while the solution for the conducting sidewalls has $\left.m_{c}=24.13, \sigma_{c}=0.123, \widetilde{R a}\right)_{c}=29.63$. This feature reflects the fact that it is the no-slip velocity condition and its associated viscous boundary layer that play a key role in controlling the dynamics of inertial convection.

\section{B. Nonlinear inertial convection: Numerical method}

As a consequence of the presence of the two no-slip sidewalls, the numerically convenient pseudo-spectral method - all the variables are expanded in Fourier series in $x$ and $y$ with the Chebyshev function in the vertical direction - cannot be adopted for the present problem. In solving the fully nonlinear problem of inertial convection, we adopt a fully three-dimensional, finite-difference method that is based on the Chorin-type projection scheme ${ }^{28}$ whose essential feature is to decouple the momentum and continuity equations. The projection scheme leads to the time discretization of the momentum and heat equations, (6) and (8), in the form

$$
\begin{gathered}
\frac{\mathbf{u}^{M}-\mathbf{u}^{n}}{\Delta t}=\widetilde{R a} \Theta^{n} \hat{\mathbf{z}}+E \nabla^{2} \mathbf{u}^{n}-\mathbf{u}^{n} \cdot \nabla \mathbf{u}^{n}-2 \hat{\mathbf{z}} \times \mathbf{u}^{n}, \\
\frac{\operatorname{Pr}}{E}\left(\frac{\Theta^{n+1}-\Theta^{n}}{\Delta t}\right)=\mathbf{u}^{n} \cdot \hat{\mathbf{z}}+\nabla^{2} \Theta^{n}-\operatorname{Pr} \mathbf{u}^{n} \cdot \nabla \Theta^{n},
\end{gathered}
$$

where $\mathbf{u}^{n}$ and $\Theta^{n}$ represent the velocity and temperature of inertial convection, respectively, at the $n$th time step with $t=t_{n}$ while $\mathbf{u}^{M}$ denotes the velocity at an intermediate time between $t_{n}$ and $t=t_{n+1}$. In the first stage of the Chorin-type projection scheme, the pressure gradient term is ignored in (39) when computing the velocity $\mathbf{u}^{M}$ at the intermediate time. In the next stage of the Chorin-type projection scheme, the velocity field $\mathbf{u}^{n+1}$ at $t=t_{n+1}$ is linked to the pressure $p^{n+1}$ by 
the equation

$$
\frac{\mathbf{u}^{n+1}-\mathbf{u}^{M}}{\Delta t}=-\nabla p^{n+1}
$$

or

$$
\mathbf{u}^{n+1}=\mathbf{u}^{M}-\Delta t \nabla p^{n+1} .
$$

Upon taking the divergence of the above equation and making use of $\nabla \cdot \mathbf{u}^{n+1}=0$, we obtain a Poisson equation governing the pressure $p^{n+1}$ at the $(n+1)$ th time step with $t=t_{n+1}$,

$$
\nabla^{2} p^{n+1}=\frac{1}{\Delta t} \nabla \cdot \mathbf{u}^{M}
$$

With the velocity $\mathbf{u}^{M}$ at the intermediate time from (39) and the pressure $p^{n+1}$ from Poisson equation (41), we can then compute the velocity field $\mathbf{u}^{n+1}$ at $t=t_{n+1}$ by using

$$
\mathbf{u}^{n+1}=\mathbf{u}^{M}-\Delta t \nabla p^{n+1} .
$$

The accuracy of our three-dimensional simulations was checked by calculating nonlinear solutions at exactly the same parameters but using different spatial and temporal resolutions.

It should be pointed out that, in addition to the boundary condition on the two sidewalls at $y=0, \Gamma$ and on the top and bottom at $z=0,1$, an additional boundary condition in the azimuthal direction, a consequence of the small-gap approximation, must be imposed for direct numerical simulation. We shall restrict our nonlinear computation to solutions that are periodic in the $x$-direction,

$$
\mathbf{u}(x, y, z)=\mathbf{u}\left(x+\Gamma_{x}, y, z\right), \quad p(x, y, z)=p\left(x+\Gamma_{x}, y, z\right), \Theta(x, y, z)=\Theta\left(x+\Gamma_{x}, y, z\right),
$$

where $\Gamma_{x}$ is a given parameter. An important factor is the size of the computational box defined by the upper and lower horizontal surfaces at $z=0,1$, by the two vertical sidewalls at $y=0, \Gamma$ and by the periodic boundary condition at $x=0, \Gamma_{x}$. On a balance between the computational cost and essential physics, we shall choose a moderately wide box with $\Gamma_{x}=\mathrm{O}\left(2 \pi / m_{c}\right)$ for our direct numerical simulation of nonlinear inertial convection.

\section{Nonlinear inertial convection: Transition to weakly turbulent states}

In comparison to the recent laboratory experiment ${ }^{18}$ focusing on the scaling law of strongly turbulent states for $(\widetilde{R a}) /(\widetilde{R a})_{c} \gg \mathrm{O}(10)$, the primary aim of our nonlinear study is at understanding weakly nonlinear states and the transition from the laminar to weakly turbulent states for the liquid metal gallium in the regime of moderately supercritical Rayleigh numbers $1<(\widetilde{R a}) /(\widetilde{R a})_{c}<\mathrm{O}(10)$. Since the thin viscous boundary layer plays an essential role in actively controlling the dynamics of inertial convection, numerical simulation must be performed longer than $t=\mathrm{O}(1 / \sqrt{E})$, which is computationally expensive for small Ekman numbers. As highlighted in the asymptotic analysis for the onset of inertial convection, the parameter range $E \leq 10^{-3}$ seems to lie in the asymptotic regime of small Ekman number. For computational reasons, we shall concentrate on the computationally less expensive case $E=10^{-3}$ for the liquid metal gallium with the Prandtl number $\operatorname{Pr}=0.023$ by performing a series of nonlinear simulation at different values of the Rayleigh numbers.

While the no-slip condition is always imposed on the bounding surface $\mathcal{S}$, our numerical simulation takes perfectly conducting condition (10) at the top and bottom and insulating condition (12) at the two sidewalls. The nonlinear simulation is guided by the key information, such as the critical Rayleigh number $(\widetilde{R a})_{c}$, provided by the onset of inertial convection. According to Table II, this particular case $-E=10^{-3}$ and $\operatorname{Pr}=0.023$ with $\Gamma=1-$ is marked by the critical azimuthal wavenumber $m_{c}=4.5298$ with the critical Rayleigh number $(\widetilde{R a})_{c}=18.284$. Consequently, we choose the three-dimensional computational domain of our nonlinear simulation confined between the no-slip upper and lower surfaces $0 \leq z \leq 1$, the two no-slip vertical sidewalls $0 \leq y \leq \Gamma=1$, and the azimuthal periodic condition with $0 \leq x \leq \Gamma_{x}=\left(2 \pi / m_{c}\right)=1.38$. 
The weakly/moderately nonlinear solution of inertial convection would be closely linked with a set of inertial wave modes that are excited and sustained by thermal instabilities. In order to elucidate the nature of a highly complicated numerical solution, we expand the velocity $\mathbf{u}$ and pressure $p$ of nonlinear inertial convection in the form

$$
\begin{aligned}
\mathbf{u} & =\widetilde{\mathbf{u}}+\sum_{k=1}^{K} \mathcal{A}_{00 k}(t) \mathbf{u}_{00 k}(y)+\frac{1}{2} \sum_{m=1}^{M} \sum_{k=1}^{K}\left[\mathcal{A}_{m 0 k}(t) \mathbf{u}_{m 0 k}(x, y)+c . c .\right] \\
& +\sum_{n=1}^{N} \sum_{k=1}^{K} \frac{1}{2}\left[\mathcal{A}_{0 n k}(t) \mathbf{u}_{0 n k}(y, z)+c . c .\right]+\sum_{m=1}^{M} \sum_{n=1}^{N} \sum_{k=1}^{K} \frac{1}{2}\left[\mathcal{A}_{m n k}(t) \mathbf{u}_{m n k}(x, y, z)+c . c .\right], \\
p & =\widetilde{p}+\sum_{k=1}^{K} \mathcal{A}_{00 k}(t) p_{00 k}(y)+\frac{1}{2} \sum_{m=1}^{M} \sum_{k=1}^{K}\left[\mathcal{A}_{m 0 k}(t) p_{m 0 k}(x, y)+c . c .\right] \\
& +\sum_{n=1}^{N} \sum_{k=1}^{K} \frac{1}{2}\left[\mathcal{A}_{0 n k}(t) p_{0 n k}(y, z)+c . c .\right]+\sum_{m=1}^{M} \sum_{n=1}^{N} \sum_{k=1}^{K} \frac{1}{2}\left[\mathcal{A}_{m n k}(t) p_{m n k}(x, y, z)+c . c .\right],
\end{aligned}
$$

where $(\widetilde{\mathbf{u}}, \widetilde{p})$ denote the viscous boundary layer, $m$ is scaled by the critical wavenumber, $\left(\mathbf{u}_{m 0 k}, p_{m 0 k}\right)$ with $m \geq 0$ and $k \geq 1$ represent the geostrophic modes, $\left(\mathbf{u}_{0 n k}, p_{0 n k}\right)$ with $n \geq 1$ and $k \geq 1$ are the axisymmetric oscillatory inertial modes, and $\left(\mathbf{u}_{m n k}, p_{m n k}\right)$ with $m \geq 1, n \geq 1$, and $k \geq 1$ denote the non-axisymmetric inertial wave modes. All the inertial modes satisfy the orthonormal condition

$$
\frac{1}{\Gamma_{c}} \int_{0}^{1} \int_{0}^{\Gamma} \int_{0}^{\Gamma_{x}}\left(\mathbf{u}_{m n k}^{*} \cdot \mathbf{u}_{m n k}\right) \mathrm{d} x \mathrm{~d} y \mathrm{~d} z=1
$$

for possible $m, n$, and $k$. In (42) and (43), the explicit expressions for $\mathbf{u}_{m n k}$ and $p_{m n k}$ can be found from Liao and Zhang ${ }^{29}$ while the coefficients, for example, $\mathcal{A}_{m n k}$ for $n \geq 1, m \geq 1$, and $k \geq 1$ at any instant $t$, can be obtained by evaluating the following integral:

$$
\mathcal{A}_{m n k}(t)=\frac{2}{\Gamma_{x}} \int_{0}^{1} \int_{0}^{\Gamma} \int_{0}^{\Gamma_{x}}\left[\left(\mathbf{u}_{m n k}\right)^{*} \cdot \mathbf{u}(x, y, z, t)\right] \mathrm{d} x \mathrm{~d} y \mathrm{~d} z .
$$

The kinetic energy density $E_{\text {kin }}(t)$ of nonlinear inertial convection at any instant $t$ can be also decomposed into different inertial modes,

$$
\begin{aligned}
E_{\mathrm{kin}}(t) & =\frac{1}{\left(\Gamma_{x} \Gamma\right)} \int_{0}^{\Gamma_{x}} \int_{0}^{\Gamma} \int_{0}^{1} \frac{1}{2}|\mathbf{u}(x, y, z, t)|^{2} \mathrm{~d} z \mathrm{~d} y \mathrm{~d} x \\
& =\frac{1}{2\left(\Gamma_{x} \Gamma\right)}\left[\sum_{k=1}^{K}\left|\mathcal{A}_{00 k}(t)\right|^{2}+\frac{1}{2} \sum_{m=1}^{M} \sum_{k=1}^{K}\left|\mathcal{A}_{m 0 k}(t)\right|^{2}+\frac{1}{2} \sum_{n=1}^{N} \sum_{k=1}^{K}\left|\mathcal{A}_{0 n k}(t)\right|^{2}\right. \\
& \left.+\frac{1}{2} \sum_{m=1}^{M} \sum_{n=1}^{N} \sum_{k=1}^{K}\left|\mathcal{A}_{m n k}^{+}(t)\right|^{2}+\frac{1}{2} \sum_{m=1}^{M} \sum_{n=1}^{N} \sum_{k=1}^{K}\left|\mathcal{A}_{m n k}^{-}(t)\right|^{2}\right]+\mathrm{O}(\sqrt{E}) .
\end{aligned}
$$

Here, a small contribution of $\mathrm{O}(\sqrt{E})$ from the thin viscous boundary layer is omitted and $K=$ $\mathrm{O}(10), M=\mathrm{O}(10)$, and $N=\mathrm{O}(10)$ are sufficient to provide an accurate description for the convective flow with moderately supercritical Rayleigh numbers. In the above decomposition, we have distinguished between the coefficient $\mathcal{A}_{m n k}^{+}(t)$ for the retrograde inertial mode and the coefficient $\mathcal{A}_{m n k}^{-}(t)$ for the prograde mode. The size and distribution of $\mathcal{A}_{m n k}^{ \pm}$offer an important insight into the nonlinear properties of inertial convection.

An interesting feature of inertial convection is that both the prograde thermal inertial wave $\left(\omega_{c}<0\right)$ and the retrograde thermal inertial wave $\left(\omega_{c}>0\right)$ have different spatial structures but with exactly the same critical Rayleigh number $(\widetilde{R a})_{c}$. It follows that the two counter-traveling inertial waves are always excited by convective instabilities and interact, depending on the phases of the two waves, destructively or constructively, resulting in a temporally oscillatory and spatially complicated convective flow even in the vicinity of the onset of inertial convection. For illustrating the two counter-traveling inertial waves, we denote $\epsilon \sim\left[(\widetilde{R a})-(\widetilde{R a})_{c}\right] /(\widetilde{R a})_{c} \ll 1$ as the amplitude of the prograde or retrograde inertial wave with the same wavenumber $m_{c}$. Upon assuming that the 
axisymmetric flow generated by the interaction of the two waves is small with the amplitude $\mathrm{O}\left(\epsilon^{2}\right)$, the leading-order convective flow, for example, the vertical flow $\hat{\mathbf{z}} \cdot \mathbf{u}$ at $z=1 / 2$, may be written in the analytical form

$$
\begin{aligned}
\hat{\mathbf{z}} \cdot \mathbf{u}(x, y, z= & \left.\frac{1}{2}, t\right)=\epsilon\left\{\sqrt{\pi^{2}+(\pi / \Gamma)^{2}+m_{c}^{2}} \sin \left(\frac{\pi y}{\Gamma}\right) \cos \left(m_{c} x\right) \sin \left(\frac{2 \pi t}{\sqrt{\pi^{2}+(\pi / \Gamma)^{2}+m_{c}^{2}}}\right)\right. \\
& +\frac{\pi^{2}}{\Gamma m_{c}} \cos \left(\frac{\pi y}{\Gamma}\right) \sin \left(m_{c} x\right) \cos \left(\frac{2 \pi t}{\sqrt{\pi^{2}+(\pi / \Gamma)^{2}+m_{c}^{2}}}\right) \\
& +\operatorname{Real}\left\{\frac { \mathrm { i } \pi ^ { 2 } } { 2 \Gamma m _ { c } } \left[\left(\mathrm{e}^{-\gamma_{c}^{+} y}-\mathrm{e}^{-\gamma_{c}^{+}(\Gamma-y)}\right) \mathrm{e}^{\mathrm{i}\left\{m_{c} x+2 \pi t / \sqrt{\pi^{2}+(\pi / \Gamma)^{2}+m_{c}^{2}}\right\}}\right.\right. \\
& \left.\left.\left.\left.+\left(\mathrm{e}^{-\gamma_{c}^{-} y}-\mathrm{e}^{-\gamma_{c}^{-}(\Gamma-y)}\right) \mathrm{e}^{\mathrm{i}\left\{m_{c} x-2 \pi t / \sqrt{\pi^{2}+(\pi / \Gamma)^{2}+m_{c}^{2}}\right.}\right\}\right]\right\}\right\}+\mathrm{O}\left(\epsilon^{2}\right),
\end{aligned}
$$

where

$$
\begin{aligned}
& \gamma_{c}^{+}=\frac{(1+\mathrm{i})}{\sqrt{E}}\left[\frac{\pi}{\sqrt{\pi^{2}+(\pi / \Gamma)^{2}+m_{c}^{2}}}\right]^{1 / 2}, \\
& \gamma_{c}^{-}=\frac{(1-\mathrm{i})}{\sqrt{E}}\left[\frac{\pi}{\sqrt{\pi^{2}+(\pi / \Gamma)^{2}+m_{c}^{2}}}\right]^{1 / 2} .
\end{aligned}
$$

It suggests that the spatial structure of even weakly nonlinear inertial convection would be quite complicated even near the threshold and that its kinetic energy would be always oscillatory with a period given approximately by $\sqrt{\pi^{2}+(\pi / \Gamma)^{2}+m_{c}^{2}}$. The profile of the oscillatory inertial convection, computed from expression (44) with $m_{c}=4.5298, E=10^{-3}, \Gamma=1$, at six different instants in the half period of oscillation, is displayed in Figure 4. Note that, if there exists only a single traveling wave at the onset of convection, the profile of the flow in a drifting frame would be typically steady near the threshold and its corresponding kinetic energy would be independent of time.

Suggested by the result of the linear stability analysis, we begin our nonlinear simulation for $E=10^{-3}, \operatorname{Pr}=0.023$, and $\Gamma=1$ at $\widetilde{R a}=18$, slightly below the onset of inertial convection. It shows that its kinetic energy $E_{\text {kin }}$, regardless of the form of initial condition used in simulation, always decays towards zero after sufficiently long integration, indicating that there exists no subcritical bifurcation for inertial convection. We then carry out the nonlinear simulation for $E=10^{-3}, \operatorname{Pr}=0.023$,

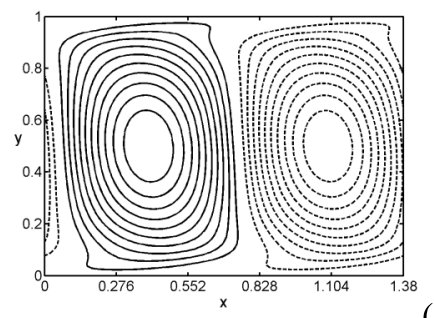

(a)
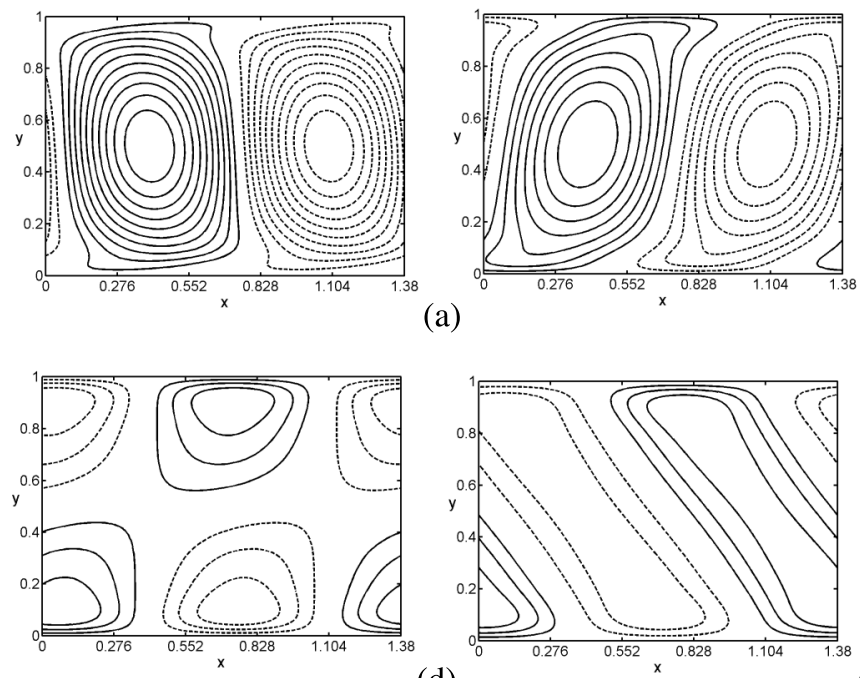

(d)

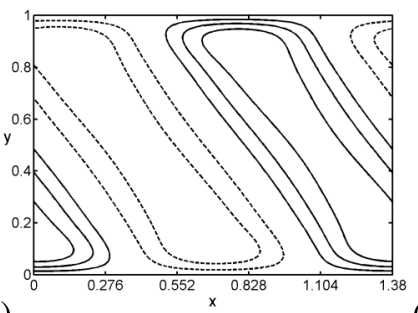

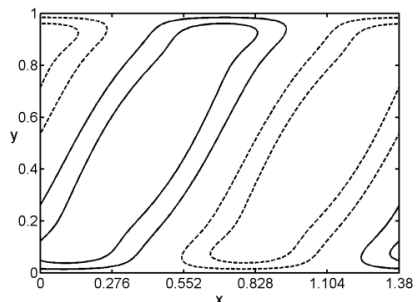

(b)

(c)

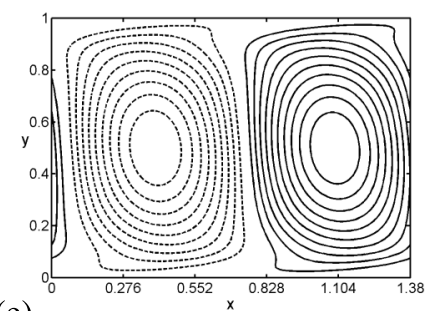

FIG. 4. Contours of the vertical flow $\hat{\mathbf{z}} \cdot \mathbf{u}$ at the middle plane $z=1 / 2$, computed from expression (44) with $m_{c}=4.5298, E=$ $10^{-3}, \Gamma=1$, at six different instants in the half period of oscillation. 

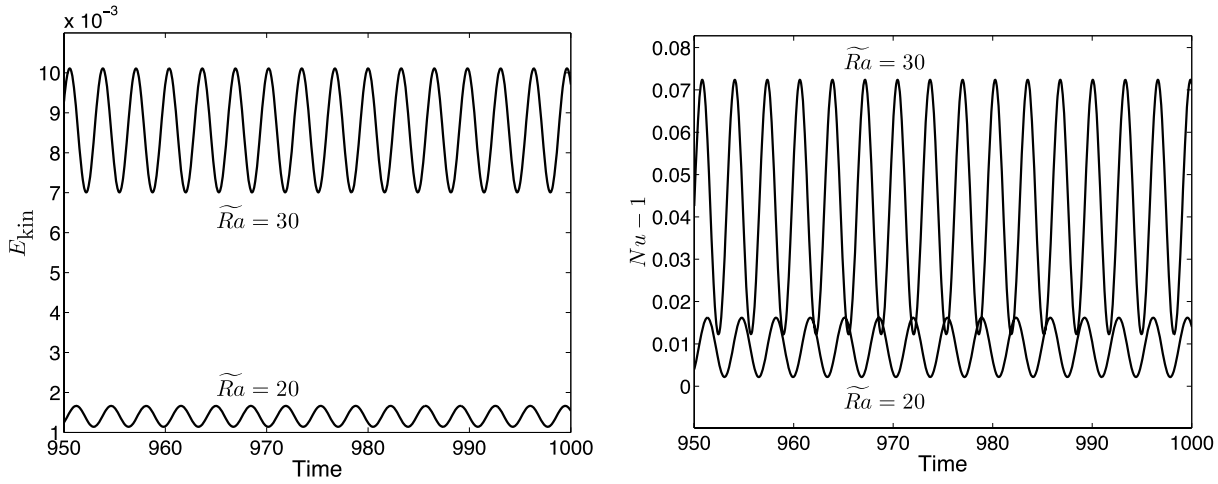

FIG. 5. Kinetic energy densities $E_{\text {kin }}$ and Nusselt numbers $(N u-1)$ of nonlinear inertial convection, obtained from direct numerical simulation using a finite difference method, are shown as a function of time for two different Rayleigh numbers, $\widetilde{R a}=20.00$ and $\widetilde{R a}=30.00$, with $E=10^{-3}, \operatorname{Pr}=0.023, \Gamma=1$. The boundary conditions are no-slip on $\mathcal{S}$ with the perfectly conducting top/bottom and the two insulating sidewalls.

and $\Gamma=1$ at $\widetilde{R a}=20.00$, which is slightly above the onset of inertial convection. To measure the amplitude of nonlinear convection, we introduce the kinetic energy density $E_{\text {kin }}$ defined as

$$
E_{\mathrm{kin}}=\frac{1}{2 \Gamma \Gamma_{x}} \int_{0}^{1} \int_{0}^{\Gamma} \int_{0}^{\Gamma_{x}}|\mathbf{u}|^{2} \mathrm{~d} x \mathrm{~d} y \mathrm{~d} z
$$

where $1 \times \Gamma \times \Gamma_{x}$ represents the dimensionless volume of the computational box. We also introduce the Nusselt number $\mathrm{Nu}$ measuring the convective heat-transport defined as

$$
N u=\frac{\text { Heat transfer with convection }}{\text { Heat transfer without convection }}=1-\frac{\operatorname{Pr}}{E \Gamma \Gamma_{x}} \int_{0}^{\Gamma} \int_{0}^{\Gamma_{x}}\left[\frac{\partial \Theta}{\partial z}\right]_{z=1} \mathrm{~d} x \mathrm{~d} y .
$$

Figure 5 shows the kinetic energy density $E_{\text {kin }}$ and the Nusselt numbers $(N u-1)$ of nonlinear convection for $\widetilde{R a}=20$ and $\widetilde{R a}=30$ as a function of time, in which transient behaviors starting from arbitrary initial conditions are not displayed. Near the threshold of inertial convection, the two most unstable modes coexist: the retrograde inertial wave is described by the critical Rayleigh number $(\widetilde{R a})_{c}=18.28$ with the critical wavenumber $m_{c}=4.53$ and the critical frequency $\omega_{c}=0.919$ while the prograde inertial mode is characterized by $(\widetilde{R a})_{c}=18.28$ with $m_{c}=4.53$ and
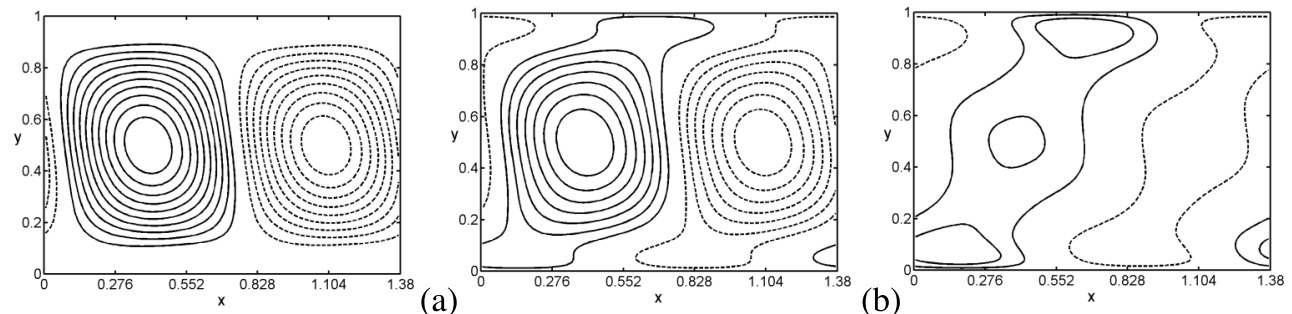

(c)
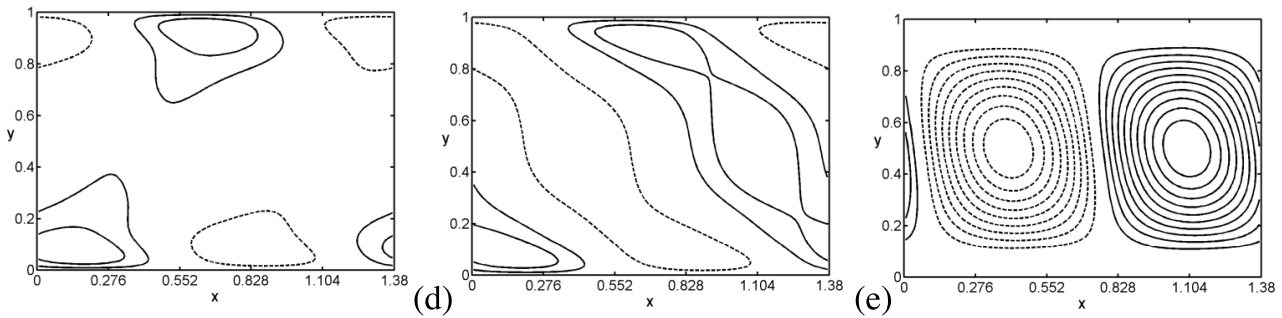

FIG. 6. Contours of the vertical flow $\hat{\mathbf{z}} \cdot \mathbf{u}$ at the middle plane $z=1 / 2$ computed from the numerical simulation of nonlinear convection at six different instants in the half period of oscillation for $E=10^{-3}, \widetilde{R a}=20.00, \operatorname{Pr}=0.023, \Gamma=1$ with the no-slip condition on $\mathcal{S}$, the perfectly conducting top/bottom, and the two insulating sidewalls. 
TABLE III. Largest four coefficients $\left|A_{m n k}\right|$ of weakly nonlinear inertial convection at two different instants - when the kinetic energy density attains a maximum $E_{\text {kin }}=1.664 \times 10^{-3}$ and a minimum $E_{\text {kin }}=1.142 \times$ $10^{-3}$ (see Figure 5) - for the weakly nonlinear oscillatory flow with $E=10^{-3}, \widetilde{R a}=20.00, \operatorname{Pr}=0.023, \Gamma=1$, where $(m, n, k)^{ \pm}$denotes the three wavenumbers of either prograde or retrograde inertial mode $\mathbf{u}_{m n k}^{ \pm}$.

\begin{tabular}{lcc}
\hline \hline At the instant $E_{\text {kin }}=\operatorname{Max}$ & $(m / 4.5298, n, k)$ & $\left|\mathcal{A}_{m n k}\right|$ \\
\hline & $(1,1,1)^{+}$ & $4.74366 \times 10^{-2}$ \\
$(1,1,1)^{-}$ & $4.72572 \times 10^{-2}$ \\
$(1,1,2)^{+}$ & $9.67532 \times 10^{-3}$ \\
$(1,1,2)^{-}$ & $8.72264 \times 10^{-3}$ \\
\hline At the instant $E_{\text {kin }}=$ Min & $(m / 4.5298, n, k)$ & $\left|\mathcal{A}_{m n k}\right|$ \\
\hline & $(1,1,1)^{+}$ & $4.73128 \times 10^{-2}$ \\
& $(1,1,1)^{-}$ & $4.71352 \times 10^{-2}$ \\
$(1,1,2)^{+}$ & $9.62975 \times 10^{-3}$ \\
& $(1,1,2)^{-}$ & $8.66241 \times 10^{-3}$ \\
\hline \hline
\end{tabular}

$\omega_{c}=-0.919$. Independent of the choice of initial condition used in direct numerical simulation, neither the retrograde inertial mode nor the prograde inertial mode can be separately physically realizable. In the weakly nonlinear regime $0<\left[\widetilde{R a}-(\widetilde{R a})_{c}\right] /(\widetilde{R a})_{c} \ll 1$, the two counter-traveling inertial waves are always convectively excited simultaneously and, hence, lead to an oscillatory convection as the primary bifurcation. The profiles of the oscillatory convection for $\widetilde{R a}=20$ from the solution of direct numerical simulation are depicted in Figure 6 at six different instants in the half period of oscillation. It can be seen that the profiles largely resemble those computed directly from analytical expression (44) and that the kinetic energy densities $E_{\text {kin }}$ in Figure 5 vary approximately with the period $\left(\sqrt{\pi^{2}+(\pi / 1.0)^{2}+(4.5298)^{2}}\right) / 2 \approx 3.4$, which is also consistent with expression (44). Note that the oscillation period of the flow is approximately $\sqrt{\pi^{2}+(\pi / 1.0)^{2}+(4.5298)^{2}} \approx 6.8$ while Figures 4 and 6 cover only its variation in a half period of the oscillation.

Furthermore, the weakly nonlinear properties of inertial convection are also reflected in its dominant coefficients $\left|A_{m n k}\right|$, which are listed in Table III after decomposing the numerical solution into the complete inertial wave modes. It reveals, as predicted by the asymptotic solution of inertial convection, that the two thermal inertial wave modes - the retrograde wave $\mathbf{u}_{m_{c} 11}^{+}$and the prograde wave $\mathbf{u}_{m_{c} 11}^{-}$with $m_{c}=4.5298$ - are always predominant. As a result of the nonlinear effects which produce a weak axisymmetric mean flow and destroy the symmetry between the retrograde mode $\mathbf{u}_{m_{c} 11}^{+}$and the prograde mode $\mathbf{u}_{m_{c} 11}^{-}$, the amplitude $\left|\mathcal{A}_{m_{c} 11}^{-}\right|=4.72572 \times 10^{-2}$ for
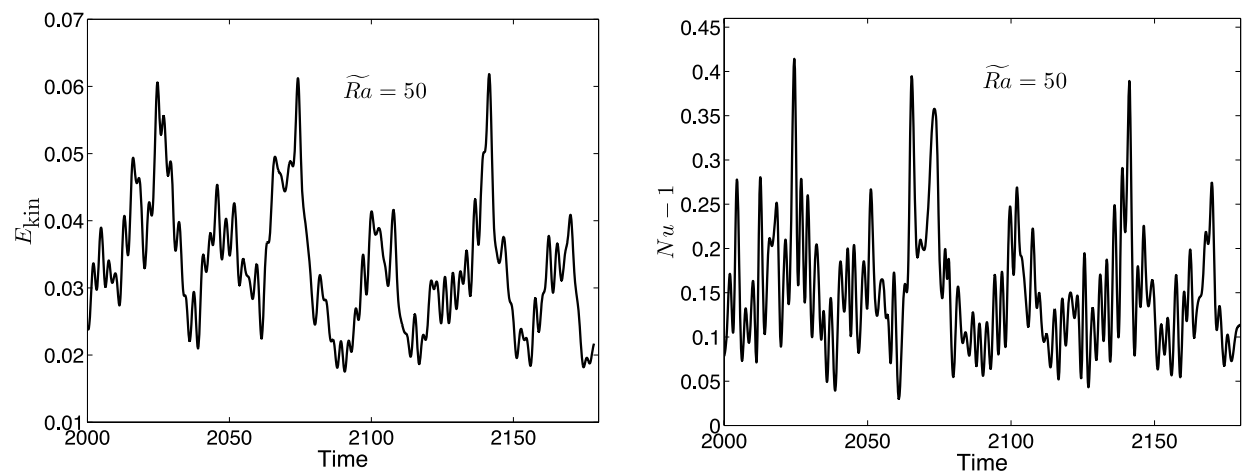

FIG. 7. Kinetic energy densities $E_{\text {kin }}$ and Nusselt numbers $(N u-1)$ of nonlinear inertial convection for the Rayleigh number $\widetilde{R a}=50.00$ with $E=10^{-3}, \operatorname{Pr}=0.023, \Gamma=1$. The boundary conditions are no-slip on $\mathcal{S}$ with the perfectly conducting top/bottom and the two insulating sidewalls. 

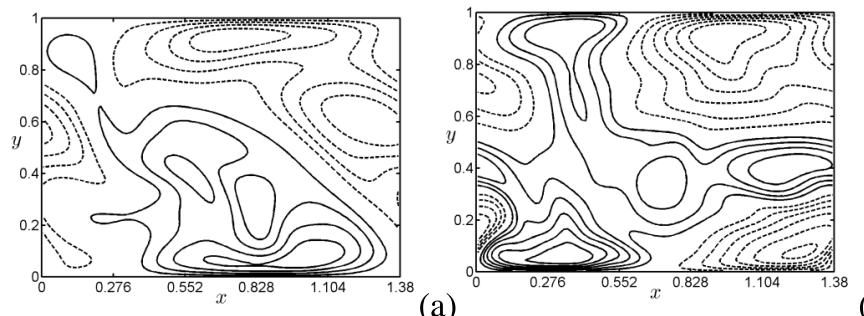

(a)

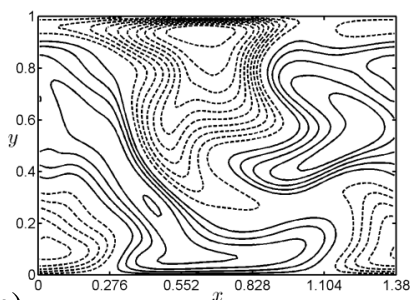

(b)

(c)
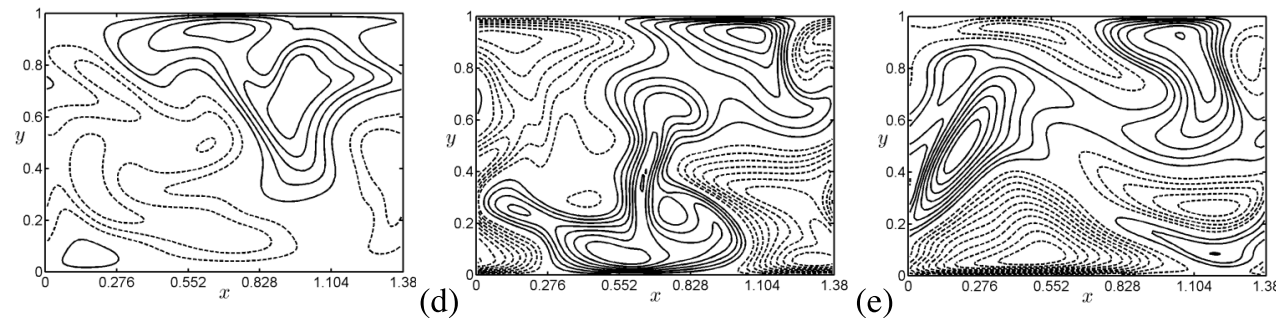

(f)

FIG. 8. Contours of $\hat{\mathbf{z}} \cdot \mathbf{u}$ at the middle plane $z=1 / 2$ at six different instants from the simulation of nonlinear convection for $E=10^{-3}, \widetilde{R a}=50.00, \operatorname{Pr}=0.023, \Gamma=1$ with the no-slip velocity condition, the perfectly conducting top/bottom, and the two insulating sidewalls.

the prograde inertial mode $\mathbf{u}_{m_{c} 11}^{+}$would be slightly different from that of the retrograde mode $\mathbf{u}_{m_{c} 11}^{-}$ with $\left|\mathcal{A}_{m_{c} 11}^{+}\right|=4.74366 \times 10^{-2}$.

As the Rayleigh number $\widetilde{R a}$ increases further, more inertial modes are convectively excited, leading to weakly turbulent inertial convection. An example of the weakly turbulent solution is

TABLE IV. Largest ten coefficients $\left|A_{m n k}\right|$ at two different instants when the kinetic energy density reaches a maximum $E_{\text {kin }}=6.122 \times 10^{-2}$ at $t=2074.4$ and a minimum $E_{\text {kin }}=1.867 \times 10^{-2}$ at $t=2116.8$ (see Figure 8) for $E=10^{-3}, \widetilde{R a}=50.00, \operatorname{Pr}=0.023, \Gamma=1$.

\begin{tabular}{ccc}
\hline \hline At the instant $E_{\text {kin }}=\operatorname{Max}$ & $(m / 4.5298, n, k)$ & $\left|\mathcal{A}_{m n k}\right|$ \\
\hline$(1,1,1)^{-}$ & $2.05386 \times 10^{-1}$ \\
$(0,1,1)$ & $1.84443 \times 10^{-1}$ \\
$(1,1,1)^{+}$ & $1.61440 \times 10^{-1}$ \\
$(1,1,4)^{-}$ & $1.00388 \times 10^{-1}$ \\
$(0,1,2)$ & $6.51191 \times 10^{-2}$ \\
$(1,1,2)^{+}$ & $6.36785 \times 10^{-2}$ \\
$(0,1,3)$ & $6.35840 \times 10^{-2}$ \\
$(1,2,1)^{+}$ & $6.14369 \times 10^{-2}$ \\
$(1,1,4)^{+}$ & $5.66571 \times 10^{-2}$ \\
$(1,2,1)^{-}$ & $5.47721 \times 10^{-2}$ \\
\hline At the instant $E_{\text {kin }}=$ Min & $(m / 4.5298, n, k)$ & $|\mathcal{A} m n k|$ \\
\hline & $(0,1,1)$ & $1.32995 \times 10^{-1}$ \\
$(1,1,1)^{-}$ & $7.97485 \times 10^{-2}$ \\
$(0,1,2)$ & $7.80425 \times 10^{-2}$ \\
$(2,1,2)^{+}$ & $5.36894 \times 10^{-2}$ \\
$(1,1,2)^{-}$ & $4.59480 \times 10^{-2}$ \\
$(1,1,2)^{+}$ & $4.57881 \times 10^{-2}$ \\
$(2,1,1)^{+}$ & $4.27939 \times 10^{-2}$ \\
$(1,1,1)^{+}$ & $4.23093 \times 10^{-2}$ \\
$(2,1,1)^{-}$ & $3.62203 \times 10^{-2}$ \\
$(1,1,4)^{-}$ & $3.52092 \times 10^{-2}$ \\
\hline \hline & & \\
\hline
\end{tabular}


presented in Figure 7 for $E=10^{-3}, P r=0.023$, and $\Gamma=1$ at $\widetilde{R a}=50$, showing the irregular temporal variation of kinetic energy densities $E_{\text {kin }}$ and Nusselt numbers $(N u-1)$ as a function of time. The corresponding irregular spatial structure of the flow is depicted in Figure 8. An insight into the weakly turbulent state can be provided by decomposing the nonlinear flow into the spectrum of inertial modes $\mathbf{u}_{m n k}$ whose coefficients $\left|A_{m n k}\right|$ are listed in Table IV at two different instants. At $t=2074.4$ in Figure 7 when its kinetic energy density attains a maximum $E_{\text {kin }}=6.122 \times 10^{-2}$, the primary inertial modes $\mathbf{u}_{m_{c} 11}^{-}$and $\mathbf{u}_{m_{c} 11}^{+}$are still dominant but the axisymmetric inertial modes such as $\mathbf{u}_{011}$ become significant. At $t=2116.8$, when the kinetic energy density reaches a minimum $E_{\text {kin }}=1.867 \times 10^{-2}$, other inertial modes such as $\mathbf{u}_{011}$ and $\mathbf{u}_{\left(2 m_{c}\right) 12}^{+}$become dominant though the prograde inertial mode $\mathbf{u}_{m_{c} 11}^{-}$is still prominent. Looking at various instants of the weakly turbulent flow, it is found that the nonlinear inertial convection is generally dominated by several inertial wave modes, $\mathbf{u}_{m_{c} 11}^{-}, \mathbf{u}_{m_{c} 11}^{+}, \mathbf{u}_{011}, \mathbf{u}_{012}$, which, convectively excited and nonlinearly interactive, appear to be responsible for the transition from the laminar oscillatory flow depicted in Figure 6 to the weakly turbulent flow in Figure 8. Comparing to other rotating convection systems, such as an infinitely extended Rayleigh-Bénard layer with the periodic boundary conditions in both the $x$ and $y$-directions, weakly nonlinear inertial convection in an annular channel represents a unique phenomenon that is marked by the nonlinear interaction of two counter-traveling inertial waves even near the threshold of convection.

\section{SUMMARY AND SOME REMARKS}

In contrast to rotating convection with large Prandtl number $P r$, inertial convection with small $\operatorname{Pr}$ breaks the rotational constraint by invoking fast oscillation through the inertial effect and is characterized by profoundly different asymptotic laws. We have studied, via both asymptotic and numerical methods, inertial convection in a Boussinesq fluid in a narrow annulus rotating rapidly about a vertical axis which can be approximately realizable in laboratory experiments. In asymptotic analysis, we have derived the asymptotic solution of inertial convection valid for $0<E \ll 1-$ which is at leading order represented by thermal inertial waves propagating in either prograde or retrograde direction and at the next order maintained by buoyancy forces against viscous damping. We have revealed that there always coexist two oppositely traveling thermal inertial waves, sustained by convection, that have the same azimuthal wavenumber, the same frequency, and the same critical Rayleigh number but different spatial structure. The numerical analysis using a Galerkin spectral method shows a quantitative agreement between the asymptotic solution for $0<E \ll 1$ and the numerical solution. We have also investigated nonlinear properties of inertial convection near the threshold through direct three-dimensional simulation using a finite-difference method based on the Chorin-type projection scheme, concentrating on the liquid metal gallium with the Prandtl number $\operatorname{Pr}=0.023$.

An objective of our nonlinear study is to understand how thermal inertial wave modes are nonlinearly interacted near the threshold of inertial convection, in attempting to elucidate the nature of weakly turbulent states in rotating convection with small Prandtl number. Our analysis suggests the following physical scenario for $0<E \ll 1$. When the Rayleigh number $\widetilde{R a}$ is sufficiently close to the critical value $(\widetilde{R a})_{c}$, the interaction of the two counter-traveling thermal inertial waves leads to a time-dependent, spatially complicated, oscillatory convective flow even in the vicinity of the threshold. When the Rayleigh number $\widetilde{R a}$ is moderately supercritical, our results indicate that the laminar to weakly turbulent transition is mainly caused by the interaction of several inertial wave modes that are excited and sustained by thermal convection. However, as suggested by Tables III and IV, there does not exist a triadic wave resonance in the nonlinear inertial convection that is primarily involved in the three inertial modes satisfying some special parametric conditions. Although our analysis suggests that the weakly turbulent state may be mathematically describable by a small number of the inertial modes, deriving an asymptotic solution of weakly nonlinear inertial convection, because of the active role played by the complex viscous boundary layers, represents a difficult and challenging theoretical task. 
Finally, it is noteworthy that the dynamics of inertial convection near the threshold and as well as in the weakly turbulent states for $1 \leq(R a) /(R a)_{c}<10$ at a fixed small $E$ is largely controlled by the effect of rotation. But the dynamics of strongly turbulent states for $(R a) /(R a)_{c} \gg \mathrm{O}(10)$ would be much more complicated, as revealed by the recent laboratory experiment, ${ }^{18}$ since in that case the stronger nonlinear effects in connection with the term $\mathbf{u} \cdot \nabla \mathbf{u}$ in (6) would help break the rotational constraint.

\section{ACKNOWLEDGMENTS}

K.Z. is supported by Leverhulme Trust Research Project Grant No. RPG-2015-096 and by Macau FDCT Grant No. 039/2013/A2; X.L. is supported by No. NSFC/11133004 and Chinese Academy of Sciences under Grant Nos. KZZD-EW-01-3 and XDB09000000. The computation made use of the high performance computing resources in the Core Facility for Advanced Research Computing at Shanghai Astronomical Observatory, Chinese Academy of Sciences.

\section{APPENDIX: THE BOUNDARY-LAYER SOLUTION $\widetilde{U}$}

An explicit solution for the boundary-layer solution $\widetilde{\mathbf{u}}$ is required for performing the asymptotic matching in the next-order analysis. Consider first the tangential component $\widetilde{\mathbf{u}}_{\text {bottom }}$ of the boundary-layer solution $\widetilde{\mathbf{u}}$ on the bottom surface at $z=0$ whose governing equation is

$$
\mathrm{i} 2 \sigma_{0} \widetilde{\mathbf{u}}_{\text {bottom }}+2 \hat{\mathbf{z}} \times \widetilde{\mathbf{u}}_{\text {bottom }}+\left(\hat{\mathbf{z}} \cdot \nabla \widetilde{p}_{\text {bottom }}\right) \hat{\mathbf{z}}=E \frac{\partial^{2} \widetilde{\mathbf{u}}_{\text {bottom }}}{\partial z^{2}},
$$

where the flow $\widetilde{\mathbf{u}}_{\text {bottom }}$ and its associated pressure $\widetilde{p}_{\text {bottom }}$ are nonzero only in the viscous boundary layer at the bottom $z=0$. The boundary-layer flow $\widetilde{\mathbf{u}}_{\text {bottom }}$ must satisfy the non-slip condition

$$
\widetilde{\mathbf{u}}_{\text {bottom }}=-\frac{1}{2}\left\{\left[\frac{\pi^{2}}{m \sigma_{0}} \sin \left(\frac{\pi y}{\Gamma}\right)-\frac{\pi}{\Gamma} \cos \left(\frac{\pi y}{\Gamma}\right)\right] \hat{\mathbf{x}}+\mathrm{i}\left[\frac{\pi^{2}+m^{2}}{m} \sin \left(\frac{\pi y}{\Gamma}\right)\right] \hat{\mathbf{y}}\right\} .
$$

Applying the operators $\hat{\mathbf{z}} \times$ and $\hat{\mathbf{z}} \times \hat{\mathbf{z}} \times$ to (A1) and combining the two resulting equations yield the fourth-order boundary-layer equation

$$
\left(\frac{\partial^{2}}{\partial \xi^{2}}-2 \mathrm{i} \sigma_{0}\right)^{2} \widetilde{\mathbf{u}}_{\text {bottom }}+4 \widetilde{\mathbf{u}}_{\text {bottom }}=0,
$$

where we have introduced a stretched boundary-layer coordinate defined by

$$
\xi=z / \sqrt{E}, \quad \frac{\partial}{\partial z} \equiv \frac{1}{\sqrt{E}} \frac{\partial}{\partial \xi} .
$$

It should be noted that $\xi=0$ is at the bottom surface while $\xi=\infty$ defines the outer edge of the boundary layer $\widetilde{\mathbf{u}}_{\text {bottom }}$ but still at the bottom surface in terms of the coordinate $z$ which does not change within the boundary layer. It can be readily shown that the boundary layer solution $\widetilde{\mathbf{u}}_{\text {bottom }}$ of (A3) satisfying both no-slip condition (A2) and the boundary-layer condition

$$
\widetilde{\mathbf{u}}_{\text {bottom }}=\frac{\partial^{2} \widetilde{\mathbf{u}}_{\text {bottom }}}{\partial \xi^{2}}=0 \text { as } \xi \rightarrow \infty
$$

can be expressed in the form

$$
\begin{aligned}
& \widetilde{\mathbf{u}}_{\text {bottom }}=\frac{1}{4}\left\{\left[\frac{\pi^{2}\left(\sigma_{0}-1\right)+m^{2} \sigma_{0}}{m \sigma_{0}} \sin \left(\frac{\pi y}{\Gamma}\right)+\frac{\pi}{\Gamma} \cos \left(\frac{\pi y}{\Gamma}\right)\right](\hat{\mathbf{x}}-\hat{\mathbf{y}} \mathrm{i}) \mathrm{e}^{-\gamma^{+} z / \sqrt{E}}\right. \\
& \left.-\left[\frac{\pi^{2}\left(\sigma_{0}+1\right)+m^{2} \sigma_{0}}{m \sigma_{0}} \sin \left(\frac{\pi y}{\Gamma}\right)-\frac{\pi}{\Gamma} \cos \left(\frac{\pi y}{\Gamma}\right)\right](\hat{\mathbf{x}}+\mathrm{i} \hat{\mathbf{y}}) \mathrm{e}^{-\gamma^{-} z / \sqrt{E}}\right\}
\end{aligned}
$$

where

$$
\gamma^{+}=(1+\mathrm{i}) \sqrt{1+\sigma_{0}}, \quad \gamma^{-}=(1-\mathrm{i}) \sqrt{1-\sigma_{0}} .
$$


Upon recognizing the vertical symmetry between the top and the bottom, the boundary-layer solution on the top surface at $z=1$, denoted by $\widetilde{\mathbf{u}}_{t o p}$, can be obtained simply by replacing $\xi=z / \sqrt{E}$ with $\xi=(1-z) / \sqrt{E}$,

$$
\begin{aligned}
& \widetilde{\mathbf{u}}_{t o p}=\frac{-1}{4}\left\{\left[\frac{\pi^{2}\left(\sigma_{0}-1\right)+m^{2} \sigma_{0}}{m \sigma_{0}} \sin \left(\frac{\pi y}{\Gamma}\right)+\frac{\pi}{\Gamma} \cos \left(\frac{\pi y}{\Gamma}\right)\right](\hat{\mathbf{x}}-\hat{\mathbf{y}} \mathrm{i}) \mathrm{e}^{-\gamma^{+}(1-z) / \sqrt{E}}\right. \\
& \left.-\left[\frac{\pi^{2}\left(\sigma_{0}+1\right)+m^{2} \sigma_{0}}{m \sigma_{0}} \sin \left(\frac{\pi y}{\Gamma}\right)-\frac{\pi}{\Gamma} \cos \left(\frac{\pi y}{\Gamma}\right)\right](\hat{\mathbf{x}}+\mathrm{i} \hat{\mathbf{y}}) \mathrm{e}^{-\gamma^{-}(1-z) / \sqrt{E}}\right\} .
\end{aligned}
$$

Evidently, the top and bottom boundary layers contribute, because of symmetry, equally to the viscous dissipation of inertial convection.

The tangential component of the viscous boundary layer $\widetilde{\mathbf{u}}$ for the outer sidewall at $y=0$, referring to as $\widetilde{\mathbf{u}}_{\text {outer }}$, and for the inner sidewall at $y=\Gamma$, referring to as $\widetilde{\mathbf{u}}_{\text {inner }}$, can be also readily derived. It can be shown that the boundary layer solution $\widetilde{\mathbf{u}}_{\text {outer }}$ is

$$
\widetilde{\mathbf{u}}_{\text {outer }}=\frac{1}{2}\left\{\left[\frac{\pi}{\Gamma} \cos (\pi z)\right] \hat{\mathbf{x}}+\mathrm{i}\left[\frac{\pi^{2}}{\Gamma m} \sin (\pi z)\right] \hat{\mathbf{z}}\right\} \mathrm{e}^{-\gamma y / \sqrt{E}},
$$

where

$$
\gamma=\sqrt{\left|\sigma_{0}\right|}\left(1+\mathrm{i} \frac{\sigma_{0}}{\left|\sigma_{0}\right|}\right)
$$

while the boundary-layer solution $\widetilde{\mathbf{u}}_{\text {inner }}$ at $y=\Gamma$ can be obtained by replacing $y / \sqrt{E}$ with $(\Gamma-$ $y) / \sqrt{E}$, which gives rise to

$$
\widetilde{\mathbf{u}}_{\text {inner }}=\frac{-1}{2}\left\{\left[\frac{\pi}{\Gamma} \cos (\pi z)\right] \hat{\mathbf{x}}+\mathrm{i}\left[\frac{\pi^{2}}{\Gamma m} \sin (\pi z)\right] \hat{\mathbf{z}}\right\} \mathrm{e}^{-\gamma(\Gamma-y) / \sqrt{E}} .
$$

It is worth mentioning that solutions (A4)-(A7) reveal explicitly that, although an oscillatory viscous boundary-layer solution always decays exponentially with the normal distance from the bounding surface $\mathcal{S}$, the thickness of the boundary layer is not generally of the order $\sqrt{E}$. For example, the thickness of the inner-wall boundary layer $\widetilde{\mathbf{u}}_{\text {inner }}$ is of the order $\left(E /\left|\sigma_{0}\right|\right)^{1 / 2}$ which is not $\mathrm{O}(\sqrt{E})$ when $0<\left|\sigma_{0}\right| \ll 1$ while the thickness of the boundary layer $\widetilde{\mathbf{u}}_{\text {bottom }}$ is of the order $\left(E /\left|1-\sigma_{0}\right|\right)^{1 / 2}$ which is not $\mathrm{O}(\sqrt{E})$ when $0<\left|1-\sigma_{0}\right| \ll 1$. This is why the classical asymptotic expansion in terms of $\sqrt{E}$ cannot be adopted in analyzing the problem of inertial convection controlled by an active, oscillatory viscous boundary layer.

${ }^{1}$ J. J. Kobine, "Inertial wave dynamics in a rotating and precessing cylinder," J. Fluid Mech. 303, 233-252 (1995).

${ }^{2} \mathrm{X}$. Liao and K. Zhang, "On flow in weakly precessing cylinders: The general asymptotic solution," J. Fluid Mech. 709, 610-621 (2012).

${ }^{3}$ Y. Lin, J. Noir, and A. Jackson, "Experimental study of fluid flows in a precessing cylindrical annulus," Phys. Fluids 26(4), 046604 (2014).

${ }^{4}$ K. Zhang, "On coupling between the Poincaré equation and the heat equation," J. Fluid Mech. 268, 211-229 (1994).

${ }^{5} \mathrm{~K}$. Zhang, "On coupling between the Poincaré equation and the heat equation: Non-slip boundary condition," J. Fluid Mech. 284, 239-256 (1995).

${ }^{6}$ F. H. Busse and R. Simitev, "Inertial convection in rotating fluid spheres," J. Fluid Mech. 498, 23-30 (2004).

${ }^{7}$ D. Cebron, M. Le Bars, J. Noir, and J. M. Aurnou, "Libration driven elliptical instability," Phys. Fluids 24, 061703 (2012).

${ }^{8}$ D. H. Kelley, S. A. Triana, D. S. Zimmerman, A. Tilgner, and D. P. Lathrop, "Inertial waves driven by differential rotation in a planetary geometry," Geophys. Astrophys. Fluid Dyn. 101, 469-487 (2007).

${ }^{9}$ M. S. Miesch, "Large-scale dynamics of the convection zone and tachocline," Living Rev. Sol. Phys. 2(1) (2005).

${ }^{10}$ S. Chandrasekhar, Hydrodynamic and Hydromagnetic Stability (Clarendon Press, Oxford, 1962).

${ }^{11}$ F. H. Busse, "Thermal instabilities in rapidly rotating systems," J. Fluid Mech. 44, 441-460 (1970).

12 P. A. Gilman, "Convection in a rotating annulus uniformly heated from below. Part 2. Nonlinear results," J. Fluid Mech. 57, 381-400 (1973).

${ }^{13}$ C. A. Jones, A. M. Soward, and A. I. Mussa, "The onset of thermal convection in a rapidly rotating sphere," J. Fluid Mech. 405, 157-179 (2000).

${ }^{14}$ K. Julien, A. M. Rubio, I. Grooms, and E. Knobloch, "Statistical and physical balances in low Rossby number RayleighBénard convection,” Geophys. Astrophys. Fluid Dyn. 106, 392-428 (2012).

15 A. M. Rubio, K. Julien, E. Knobloch, and J. B. Weiss, "Upscale energy transfer in three-dimensional rapidly rotating turbulent convection," Phys. Rev. Lett. 112(14), 144501 (2014).

${ }^{16} \mathrm{~K}$. Zhang and P. H. Roberts, "Thermal inertial waves in a rotating fluid layer: Exact and asymptotic solutions," Phys. Fluids 9, 1980-1987 (1997). 
${ }^{17}$ J. M. Aurnou and P. L. Olson, "Experiments on Rayleigh-Bénard convection, magnetoconvection and rotating magnetoconvection in liquid gallium," J. Fluid Mech. 430, 283-307 (2001).

${ }^{18}$ E. M. King and J. M. Aurnou, "Turbulent convection in liquid metal with and without rotation," Proc. Natl. Acad. Sci. U. S. A. 110, 6688-6693 (2013)

${ }^{19}$ A. Ribeiro, G. Fabre, J.-L. Guermond, and J. M. Aurnou, "Canonical models of geophysical and astrophysical flows: Turbulent convection experiments in liquid metals," Metals 5, 289-335 (2015).

${ }^{20}$ R. P. Davies-Jones and P. A. Gilman, "Convection in a rotating annulus uniformly heated from below," J. Fluid Mech. 46, 65-81 (1971).

${ }^{21}$ F. H. Busse, "Convection in a narrow annular channel rotating about its axis of symmetry," J. Fluid Mech. 537, 145-154 (2005).

${ }^{22}$ X. Liao, K. Zhang, and Y. Chang, "Convection in rotating annular channels heated from below: Part 1. Linear stability and weakly nonlinear mean flows," Geophys. Astrophys. Fluid Dyn. 99, 445-465 (2005)

${ }^{23}$ Y. Chang, X. Liao, and K. Zhang, "Convection in rotating annular channels heated from below. Part 2. Transition to turbulence," Geophys. Astrophys. Fluid Dyn. 100, 215-241 (2006).

${ }^{24} \mathrm{~L}$. Li, X. Liao, and K. Zhang, “Countertraveling waves in rotating Rayleigh-Bénard convection,” Phys. Rev. E 77, 027301 (2008).

${ }^{25}$ Z. Cui, K. Zhang, and X. Liao, "On the completeness of inertial wave modes in rotating annular channels," Geophys. Astrophys. Fluid Dyn. 108, 44-59 (2014).

${ }^{26}$ H. P. Greenspan, The Theory of Rotating Fluids (Cambridge University Press, 1968).

${ }^{27} \mathrm{~K}$. Zhang and X. Liao, "On the initial value problem in a rotating circular cylinder," J. Fluid Mech. 610, 425-443 (2008).

${ }^{28}$ A. J. Chorin, "Numerical solutions of Navier-Stokes equations," Math. Comput. 22, 745-762 (1968).

${ }^{29}$ X. Liao and K. Zhang, "Inertial oscillation, inertial wave and initial value problem in rotating annular channels," Geophys. Astrophys. Fluid Dyn. 103, 199-222 (2009). 